ESAIM: COCV 27 (2021) 78

https://doi.org/10.1051/cocv/2021073
ESAIM: Control, Optimisation and Calculus of Variations

www.esaim-cocv.org

\title{
ON THE UNIFORM CONTROLLABILITY FOR A FAMILY OF NON-VISCOUS AND VISCOUS BURGERS- $\alpha$ SYSTEMS
}

\author{
Raul K.C. Araújo ${ }^{1, *}$, Enrique Fernández-Cara ${ }^{2, * *}$ \\ AND Diego A. SouZA ${ }^{2, * *, * * *}$ (])
}

\begin{abstract}
In this paper we study the global controllability of families of the so called non-viscous and viscous Burgers- $\alpha$ systems by using boundary and space independent distributed controls. In these equations, the usual convective velocity of the Burgers equation is replaced by a regularized velocity, induced by a Helmholtz filter of characteristic wavelength $\alpha$. First, we prove a global exact controllability result (uniform with respect to $\alpha$ ) for the non-viscous Burgers- $\alpha$ system, using the return method and a fixed-point argument. Then, the global uniform exact controllability to constant states is deduced for the viscous equations. To this purpose, we first prove a local exact controllability property and, then, we establish a global approximate controllability result for smooth initial and target states.
\end{abstract}

Mathematics Subject Classification. 93B05, 35Q35, 35G25.

Received March 5, 2021. Accepted June 24, 2021.

\section{INTRODUCTION}

Let $L>0$ and $T>0$ be given. Let us present the notations used along this work. The symbols $C, \widehat{C}$ and $C_{i}, i=0,1, \ldots$ stand for positive constants (usually depending on $L$ and $T$ and independent of $\alpha$ ). For any $r \in[1,+\infty]$ and any given Banach space $X,\|\cdot\|_{L^{r}(X)}$ will stand for the usual norm in Lebesgue-Bochner space $L^{r}(0, T ; X)$. In particular, the norms in $L^{r}(0, L), L^{r}(0, T)$ and $L^{r}((0, T) \times(0, L))$ will be denoted by $\|\cdot\|_{r}$. In this paper, we will consider the following two families of controlled systems:

$$
\begin{cases}y_{t}+z y_{x}=p(t) & \text { in }[0, T] \times[0, L], \\ z-\alpha^{2} z_{x x}=y & \text { in }[0, T] \times[0, L], \\ z(\cdot, 0)=v_{l}, \quad z(\cdot, L)=v_{r} & \text { in }[0, T], \\ y(\cdot, 0)=v_{l} & \text { in } I_{l}, \\ y(\cdot, L)=v_{r} & \text { in } I_{r}, \\ y(0, \cdot)=y_{0} & \text { in }[0, L],\end{cases}
$$

* Partially supported by CNPq (Brazil).

** Partially supported by grant MTM2016-76990-P, Ministry of Economy and Competitiveness (Spain).

Keywords and phrases: Burgers- $\alpha$ system, global exact controllability, return method.

${ }^{1}$ Department of Mathematics, Federal University of Pernambuco, UFPE, CEP 50740-545, Recife, PE, Brazil.

2 University of Sevilla, Dpto. E.D.A.N, Aptdo 1160, 41080 Sevilla, Spain.

*** Corresponding author: desouza@us.es

(C) The authors. Published by EDP Sciences, SMAI 2021

This is an Open Access article distributed under the terms of the Creative Commons Attribution License (https://creativecommons.org/licenses/by/4.0), which permits unrestricted use, distribution, and reproduction in any medium, provided the original work is properly cited. 
where $I_{l}=\left\{t \in[0, T]: v_{l}(t)>0\right\}$ and $I_{r}=\left\{t \in[0, T]: v_{r}(t)<0\right\}$, and

$$
\left\{\begin{array}{lll}
y_{t}-\mu y_{x x}+z y_{x}=p(t) & \text { in } & (0, T) \times(0, L), \\
z-\alpha^{2} z_{x x}=y & \text { in } & (0, T) \times(0, L), \\
z(\cdot, 0)=y(\cdot, 0)=v_{l} & \text { in } & (0, T), \\
z(\cdot, L)=y(\cdot, L)=v_{r} & \text { in } & (0, T), \\
y(0, \cdot)=y_{0} & \text { in } & (0, L) .
\end{array}\right.
$$

These are the so called non-viscous and viscous convectively filtered Burgers equations (also known in the literature as the Burgers- $\alpha$ system or Leray-Burgers equation). The couples $(y, z)$ and the triplets $\left(p, v_{l}, v_{r}\right)$ stand for the corresponding states and controls. The parameter $\mu>0$ is the fluid viscosity and $\alpha>0$ is the characteristic wavelength of the considered Helmholtz filter. For simplicity, throughout this paper we will take $\mu=1$. All the results can be extended without difficulty to the case where $\mu$ is an arbitrary positive number.

Obviously, (1.1) and (1.2) can be regarded as nonlinear regularizations of the non-viscous and viscous Burgers equations. These systems and some related variants have already been studied. More precisely, (1.2) is the $b=0$ case of the $b$-family:

$$
\left\{\begin{array}{l}
y_{t}-\mu y_{x x}+z y_{x}+b z_{x} y=p(t) \\
z-\alpha^{2} z_{x x}=y
\end{array}\right.
$$

or, equivalently,

$$
z_{t}-\alpha^{2} z_{x x t}-\mu z_{x x}+\mu \alpha^{2} z_{x x x x}+(b+1) z z_{x}-\alpha^{2} z z_{x x x}-b \alpha^{2} z_{x} z_{x x}=p(t)
$$

It has been studied in [37] as a model for the nonlinear 1D wave dynamics in a fluid including the effects of convection and stretching; the dimensionless parameter $b$ measures the relative strength of these effects and $z$ can be viewed as the fluid velocity in the $x$ direction (or equivalently the height of the free surface of the fluid above a flat bottom). When $b=2,(1.3)$ is the so-called viscous Camassa-Holm equation; it describes the unidirectional surface waves at a free surface of shallow water under the influence of gravity, see [10]. When $b=3$, we are dealing with the viscous Degasperis-Procesi equation, which plays a similar role in water wave theory, see [19].

It is interesting to highlight that this regularization idea was first employed by Leray in [39] to prove the existence of a solution to the Navier-Stokes equations. It has been used to capture shocks in the Burgers equation in $[5,7,8,42,43]$. It has also been employed in other contexts, such as the analysis of compressible Euler equations, scalar conservations laws and aggregation equations, see [4, 6, 9, 18, 46]. Finally, systems like (1.1) and (1.2) can also be viewed as simplified 1D versions of the so called Leray- $\alpha$ system introduced some time ago to describe turbulent flows, as an alternative to the classical averaged Reynolds models; see [12, 29, 30].

Our main results deal with the global uniform (with respect to $\alpha$ ) exact controllability of (1.1) and (1.2). More precisely, one has:

Theorem 1.1. Let $\alpha>0$ be given. The non-viscous Burgers- $\alpha$ system (1.1) is globally exactly controllable in $C^{1}$. That is, for any given $y_{0}, y_{T} \in C^{1}([0, L])$, there exist a space-independent control $p^{\alpha} \in C^{0}([0, T]), a$ couple of boundary controls $\left(v_{l}^{\alpha}, v_{r}^{\alpha}\right) \in C^{1}\left([0, T] ; \mathbb{R}^{2}\right)$ and an associated state pair $\left(y^{\alpha}, z^{\alpha}\right) \in C^{1}\left([0, T] \times[0, L] ; \mathbb{R}^{2}\right)$ satisfying (1.1) and

$$
y^{\alpha}(T, \cdot)=y_{T} \quad \text { in } \quad(0, L) .
$$


Moreover, there exists a positive constant $C>0$ (depending on $L, T, y_{0}$ and $y_{T}$ but independent of $\alpha$ ) such that

$$
\left\|\left(z^{\alpha}, y^{\alpha}\right)\right\|_{C^{1}\left([0, T] \times[0, L] ; \mathbb{R}^{2}\right)}+\left\|p^{\alpha}\right\|_{C^{0}([0, T])}+\left\|\left(v_{l}^{\alpha}, v_{r}^{\alpha}\right)\right\|_{C^{1}\left([0, T] ; \mathbb{R}^{2}\right)} \leq C .
$$

Theorem 1.2. Let $\alpha>0$ be given. The viscous Burgers- $\alpha$ system (1.2) is globally exactly controllable in $L^{\infty}$ to constant trajectories. That is, for any given $y_{0} \in L^{\infty}(0, L)$ and $N \in \mathbb{R}$, there exist a space-independent control $p^{\alpha} \in C^{0}([0, T])$, a couple of boundary controls $\left(v_{l}^{\alpha}, v_{r}^{\alpha}\right) \in H^{3 / 4}\left(0, T ; \mathbb{R}^{2}\right)$ and an associated state pair $\left(y^{\alpha}, z^{\alpha}\right) \in L^{2}\left(0, T ; H^{1}\left(0, L ; \mathbb{R}^{2}\right)\right) \cap L^{\infty}\left(0, T ; L^{\infty}\left(0, L ; \mathbb{R}^{2}\right)\right)$ satisfying $(1.2)$,

$$
y^{\alpha}(T, \cdot)=N \quad \text { in } \quad(0, L)
$$

and the following estimate

$$
\left\|p^{\alpha}\right\|_{C^{0}([0, T])}+\left\|\left(v_{l}^{\alpha}, v_{r}^{\alpha}\right)\right\|_{H^{3 / 4}\left([0, T] ; \mathbb{R}^{2}\right)} \leq C
$$

where $C$ is a positive constant (depending on $L, T, y_{0}$ and $N$ but independent of $\alpha$ ). Moreover, if $y_{0} \in H_{0}^{1}(0, L)$ then the same conclusion holds with

$$
\left(y^{\alpha}, z^{\alpha}\right) \in L^{2}\left(0, T ; H^{2}\left(0, L ; \mathbb{R}^{2}\right)\right) \cap H^{1}\left(0, T ; L^{2}\left(0, L ; \mathbb{R}^{2}\right)\right) .
$$

In this paper, we are going to deal with situations that lead to new difficulties compared to previous works on nonlinear parabolic equations. Let us explain these differences:

- Nonlocal nonlinearities. In (1.1) and (1.2), the usual convective term is replaced and a filtered (averaged) velocity appears. As a consequence, the arguments in [11] must be modified, as shown below.

- Uniform controllability. Performing careful estimates of the controls, global uniform controllability results can be obtained. This way, we will be able to generalize previous control results for nonlinear parabolic equations with nonlocal nonlinearities, see $[11,41]$.

For completeness, let us mention some previous works on the well-posedness and control of the previous systems and other similar models. Global and local well-posedness are respectively established in [23] (when the boundary conditions are homogeneous) and [13, 45]. Moreover, there are many important works dealing with the controllability properties of parabolic equations and systems (see $[22,25,28,31]$ ) and non-viscous and viscous Burgers equations (see [11, 17, 20, 26, 31, 33, 34, 38, 41, 44]). In the case of the Burgers- $\alpha$ system, the local uniform null controllability of the viscous system (1.2) with distributed and boundary controls was studied in [1]; later, the results have been extended to any equation of the $b$-family in [27]. In higher dimensions, local uniform (distributed and boundary) null controlability results for the Leray- $\alpha$ system have been obtained in [2].

The rest of this paper is organized as follows. In Section 2, we prove some results concerning the existence, uniqueness and regularity of the solutions. Sections 3 and 4 deal with the proofs of Theorems 1.1 and 1.2. Finally, in Section 5, we present several additional comments and questions.

\section{Preliminaries}

\subsection{Notations and classical results}

Let us denote by $C_{b}^{0}(\mathbb{R})$ (resp. $C_{b}^{0,1}(\mathbb{R})$ ) the Banach space of bounded continuous functions on $\mathbb{R}$ (resp. bounded Lipschitz-continuous functions on $\mathbb{R})$. Let $C_{x}^{0,1}([0, T] \times \mathbb{R})$ be the space of functions $f$ : $[0, T] \times \mathbb{R} \mapsto \mathbb{R}$ that are continuous in $x$ and $t$ and globally Lipschitz-continuous in space, with a Lipschitz constant independent of $t$. 
In the sequel, for any given $f \in C^{0}([0, T] \times \mathbb{R})$, the associated flux function $\Phi=\Phi(s ; t, x)$ is defined as follows:

$$
\left\{\begin{array}{l}
\frac{\partial \Phi}{\partial t}(s ; t, x)=f(t, \Phi(s ; t, x)) \\
\Phi(s ; s, x)=x
\end{array}\right.
$$

The mapping $\Phi$ contains all the information on the trajectories of the particles transported by the velocity $f$. Furthermore, we have the following existence, uniqueness and regularity result:

Proposition 2.1 ([21], Thm. 10.19). Assume that $f \in C_{x}^{0,1}([0, T] \times \mathbb{R})$ and $f_{x}$ belongs to the space $C^{0}([0, T] \times \mathbb{R})$. Then, there exists a unique flux function associated to $f$, that is, a unique function $\Phi:[0, T] \times[0, T] \times \mathbb{R} \mapsto \mathbb{R}$ satisfying (2.1) for all $(s, t, x) \in[0, T] \times[0, T] \times \mathbb{R}$. Moreover, $\Phi \in C^{1}([0, T] \times[0, T] \times \mathbb{R})$.

See $[35,36]$ for other similar results. Under the assumptions in Proposition 2.1, it is well known that for any $s, t \in[0, T]$ the mapping $\Phi(s ; t, \cdot): \mathbb{R} \mapsto \mathbb{R}$ is a diffeomorphism, with

$$
\Phi(s ; t, \cdot)^{-1}=\Phi(t ; s, \cdot) .
$$

Let us now recall a result from Bardos and Frisch [3]. To this purpose, let us first note that, for any given Banach space $X$ with norm $\|\cdot\|_{X}$ and any function $u \in C^{1}([0, T] ; X)$, the following inequality holds:

$$
\frac{\mathrm{d}}{\mathrm{d} t^{+}}\|u(t)\|_{X} \leq\left\|u_{t}(t)\right\|_{X} \quad \text { in }(0, T)
$$

where $\mathrm{d} / \mathrm{d} t^{+}$represents the time derivative to the right.

Proposition 2.2 ([3], Lem. 1). Let the velocity $v$ and the source $g$ be given respectively in the spaces $C^{0}\left([0, T] ; C_{b}^{0,1}(\mathbb{R})\right) \cap C_{x}^{0,1}([0, T] \times \mathbb{R})$ and $C^{0}\left([0, T] ; C_{b}^{0}(\mathbb{R})\right)$. Then, any solution in the space $C^{0}\left([0, T] ; C_{b}^{1}(\mathbb{R})\right) \cap$ $C^{1}\left([0, T] ; C_{b}^{0}(\mathbb{R})\right)$ to the equation

$$
y_{t}+v y_{x}=g \text { in }(0, T) \times \mathbb{R}
$$

satisfies the following inequality

$$
\frac{\mathrm{d}}{\mathrm{d} t^{+}}\|y(t, \cdot)\|_{C_{b}^{0}(\mathbb{R})} \leq\|g(t, \cdot)\|_{C_{b}^{0}(\mathbb{R})} \quad \text { in }(0, T) .
$$

Proof. Let $\Phi$ be the flow associated to $v$. For any $(s, t, x) \in[0, T] \times[0, T] \times \mathbb{R}$, we have by $(2.3)$ that

$$
\frac{\mathrm{d}}{\mathrm{d} t} y(t, \Phi(s ; t, x))=g(t, \Phi(s ; t, x)) .
$$

Using this identity and the fact that $\Phi(s ; t, \cdot)$ is a diffeomorphism, we get:

$$
\left\|\frac{\mathrm{d}}{\mathrm{d} t} y(t, \Phi(s ; t, \cdot))\right\|_{C_{b}^{0}(\mathbb{R})} \leq\|g(t, \cdot)\|_{C_{b}^{0}(\mathbb{R})} .
$$

Now, the result follows easily from this and from (2.2).

The last result of this section is an immediate consequence of Banach's Fixed-Point Theorem: 
Theorem 2.3. Let $\left(E,\|\cdot\|_{E}\right)$ and $\left(F,\|\cdot\|_{F}\right)$ be Banach spaces, with $F$ continuously embedded in $E$. Let $B$ be a subset of $F$ and let $G: B \mapsto B$ be a mapping such that

$$
\|G(u)-G(v)\|_{E} \leq \gamma\|u-v\|_{E} \quad \forall u, v \in B, \text { for some } \gamma \in(0,1)
$$

Let us denote by $\widetilde{B}$ the closure of $B$ for the norm $\|\cdot\|_{E}$. Then, $G$ can be uniquely extended to a continuous mapping $\widetilde{G}: \widetilde{B} \mapsto \widetilde{B}$ that possesses a unique fixed-point in $\widetilde{B}$.

\subsection{Well-posedness of the viscous burgers- $\alpha$ system}

Let us introduce the Hilbert space $E:=H^{3 / 4}(0, T) \times H^{3 / 4}(0, T)$. It is not difficult to check that the trace operator $\Gamma: L^{2}\left(0, T ; H^{2}(0, L)\right) \cap H^{1}\left(0, T ; L^{2}(0, L)\right) \mapsto E$, defined by $\Gamma(\xi):=(\xi(\cdot, 0), \xi(\cdot, L))$, is surjective, see [40], p. 18. Furthermore, there exists a linear continuous mapping $S: E \mapsto L^{2}\left(0, T ; H^{2}(0, L)\right) \cap H^{1}\left(0, T ; L^{2}(0, L)\right)$ such that $\Gamma \circ S=I_{E}$. Thus, for each $\left(v_{l}, v_{r}\right) \in E$, we can get $\xi \in L^{2}\left(0, T ; H^{2}(0, L)\right) \cap H^{1}\left(0, T ; L^{2}(0, L)\right)$ such that

$$
\|\xi\|_{L^{2}\left(H^{2}\right) \cap H^{1}\left(L^{2}\right)} \leq C\left(\left\|v_{l}\right\|_{H^{3 / 4}}+\left\|v_{r}\right\|_{H^{3 / 4}}\right),
$$

for some $C>0$.

The following result concerns the global existence and uniqueness of a solution to the viscous Burgers- $\alpha$ system:

Proposition 2.4. Let $\alpha>0, f \in L^{\infty}\left(0, T ; L^{\infty}(0, L)\right), y_{0} \in H^{1}(0, L)$ and $v_{l}, v_{r} \in H^{3 / 4}(0, T)$ be given. Assume that the following compatibility relations hold:

$$
v_{l}(0)=y_{0}(0) \quad \text { and } \quad v_{r}(0)=y_{0}(L)
$$

Then there exists a unique solution $\left(y^{\alpha}, z^{\alpha}\right)$ to the Burgers- $\alpha$ system:

$$
\left\{\begin{array}{lll}
y_{t}^{\alpha}-y_{x x}^{\alpha}+z^{\alpha} y_{x}^{\alpha}=f & \text { in } & (0, T) \times(0, L), \\
z^{\alpha}-\alpha^{2} z_{x x}^{\alpha}=y^{\alpha} & \text { in } & (0, T) \times(0, L), \\
z^{\alpha}(\cdot, 0)=y^{\alpha}(\cdot, 0)=v_{l} & \text { in } & (0, T) \\
z^{\alpha}(\cdot, L)=y^{\alpha}(\cdot, L)=v_{r} & \text { in } & (0, T), \\
y^{\alpha}(0, \cdot)=y_{0} & \text { in } & (0, L) .
\end{array}\right.
$$

with

$$
\left\{\begin{array}{l}
y^{\alpha} \in H^{1}\left(0, T ; L^{2}(0, L)\right) \cap L^{2}\left(0, T ; H^{2}(0, L)\right) \cap C^{0}\left([0, T] ; H^{1}(0, L)\right), \\
z^{\alpha} \in H^{1}\left(0, T ; L^{2}(0, L)\right) \cap L^{2}\left(0, T ; H^{4}(0, L)\right) \cap C^{0}\left([0, T] ; H^{3}(0, L)\right) .
\end{array}\right.
$$

Let us set $M_{T}:=\left\|y^{0}\right\|_{\infty}+\left\|v_{l}\right\|_{\infty}+\left\|v_{r}\right\|_{\infty}+T\|f\|_{\infty}$. Then, the following estimates holds:

$$
\left\{\begin{array}{l}
\left\|y^{\alpha}\right\|_{\infty} \leq M_{T}, \quad\left\|z^{\alpha}\right\|_{\infty} \leq M_{T}, \\
\left\|y^{\alpha}\right\|_{H^{1}\left(L^{2}\right) \cap L^{2}\left(H^{2}\right)}+\left\|y^{\alpha}\right\|_{L^{\infty}\left(H^{1}\right)} \leq C e^{C M_{T}^{2}}\left(\|f\|_{2}+\left\|y_{0}\right\|_{H^{1}}+\left\|v_{l}\right\|_{H^{3 / 4}}+\left\|v_{r}\right\|_{H^{3 / 4}}\right), \\
\left\|z^{\alpha}\right\|_{2}+\alpha\left\|z_{x}^{\alpha}\right\|_{2}+\alpha^{2}\left\|z_{x x}^{\alpha}\right\|_{2} \leq C e^{C M_{T}^{2}}\left[\|f\|_{2}+\left\|y_{0}\right\|_{H^{1}}+\left(1+\alpha^{2}\right)\left\|\left(v_{l}, v_{r}\right)\right\|_{H^{3 / 4} \times H^{3 / 4}}\right]
\end{array}\right.
$$

where the constants $C$ do not depend of $\alpha$. 
Proof. The proof of existence can be reduced to find a fixed-point of an appropriate mapping $\Lambda_{\alpha}$. Thus, note first that there exists $\xi \in L^{2}\left(0, T ; H^{2}(0, L)\right) \cap H^{1}\left(0, T ; L^{2}(0, L)\right)$ with

$$
\xi(\cdot, 0)=v_{l}, \quad \xi(\cdot, L)=v_{r} \text { on }(0, T) \text { and } \xi(0, \cdot)=y_{0}, \quad \text { in }(0, L) .
$$

Accordingly, for each $\bar{y} \in L^{\infty}\left(0, T ; L^{\infty}(0, L)\right)$ there exists exactly one $z \in L^{\infty}\left(0, T ; H^{2}(0, L)\right)$ with

$$
\begin{cases}z-\alpha^{2} z_{x x}=\bar{y} & \text { in } \quad(0, T) \times(0, L) \\ z(\cdot, 0)=v_{l}, z(\cdot, L)=v_{r} & \text { in } \quad(0, T)\end{cases}
$$

and, moreover, using a lifting argument together with standard energy estimates for elliptic equations, we get a positive constant $C$ (independent of $\alpha$ ) such that:

$$
\begin{aligned}
& \|z\|_{2}^{2}+2 \alpha^{2}\left\|z_{x}\right\|_{2}^{2}+\alpha^{4}\left\|z_{x x}\right\|_{2}^{2} \leq C\left(\|\bar{y}\|_{2}^{2}+\|\xi\|_{2}^{2}+\alpha^{2}\left\|\xi_{x}\right\|_{2}^{2}+\alpha^{4}\left\|\xi_{x x}\right\|_{2}^{2}\right) \\
& \|z\|_{L^{\infty}\left(L^{\infty}\right)} \leq\|\bar{y}\|_{L^{\infty}\left(L^{\infty}\right)}+\left\|v_{l}\right\|_{\infty}+\left\|v_{r}\right\|_{\infty} .
\end{aligned}
$$

With this $z$, by applying (for instance) the Faedo-Galerkin method, we can easily prove the existence of a solution $y$ to the linear parabolic equation

$$
\begin{cases}y_{t}-y_{x x}+z y_{x}=f & \text { in } \quad(0, T) \times(0, L) \\ y(\cdot, 0)=v_{l}, y(\cdot, L)=v_{r} & \text { in } \quad(0, T) \\ y(0, \cdot)=y_{0} & \text { in } \quad(0, L)\end{cases}
$$

that satisfies

$$
y \in H^{1}\left(0, T ; L^{2}(0, L)\right) \cap L^{2}\left(0, T ; H^{2}(0, L)\right) \cap C^{0}\left([0, T] ; H^{1}(0, L)\right)
$$

and

$$
\left\|y_{t}\right\|_{L^{2}\left(L^{2}\right)}+\|y\|_{L^{2}\left(H^{2}\right)}+\|y\|_{L^{\infty}\left(H^{1}\right)} \leq C\left(\left\|y_{0}\right\|_{H^{1}}+\|f\|_{L^{2}\left(L^{2}\right)}+\left\|v_{l}\right\|_{H^{3 / 4}}+\left\|v_{r}\right\|_{H^{3 / 4}}\right) e^{C\|z\|_{\infty}^{2}},
$$

for some $C>0$ that is independent of $\alpha$.

Arguing as in the proof of Lemma 1 in [1], we can deduce that the solution to (2.7) belongs to the space $C^{0}\left([0, T] ; H^{1}(0, L)\right)$ and, in particular, $\|y\|_{L^{\infty}\left(L^{\infty}\right)} \leq M_{T}$. Accordingly, we can introduce the bounded closed convex set

$$
K:=\left\{\bar{y} \in L^{\infty}\left(0, T ; L^{\infty}(0, L)\right):\|\bar{y}\|_{L^{\infty}\left(L^{\infty}\right)} \leq M_{T}\right\}
$$

and the mapping $\Lambda_{\alpha}: K \mapsto K$, with $\Lambda_{\alpha}(\bar{y})=y$. Obviously, $\Lambda_{\alpha}$ is well-defined and continuous and, moreover, we can see from the estimates in (2.8) that $G:=\Lambda_{\alpha}(K)$ is bounded in $L^{\infty}\left(0, T ; H^{1}(0, L)\right)$ and $G_{t}:=\left\{u_{t} ; u \in G\right\}$ is bounded in $L^{2}\left(0, T ; L^{2}(0, L)\right)$. From classical results of the Aubin-Lions kind (see [47]), we deduce that $G$ is relatively compact in $L^{\infty}\left(0, T ; L^{\infty}(0, L)\right)$. Therefore, by Schauder's Fixed Point Theorem, $\Lambda_{\alpha}$ has a fixed point in $K$, which obviously implies the existence of a solution to (2.4).

We prove now that the solution is unique. Let $\left(y^{\alpha}, z^{\alpha}\right)$ and $\left(\widehat{y}^{\alpha}, \widehat{z}^{\alpha}\right)$ be two solutions to (2.4) and let us introduce $u:=y^{\alpha}-\widehat{y}^{\alpha}$ and $v:=z^{\alpha}-\widehat{z}^{\alpha}$. Then,

$$
\begin{cases}u_{t}-u_{x x}+z^{\alpha} u_{x}=-v \widehat{y}_{x}^{\alpha} & \text { in }(0, T) \times(0, L), \\ v-\alpha^{2} v_{x x}=u & \text { in }(0, T) \times(0, L), \\ u(\cdot, 0)=u(\cdot, L)=v(\cdot, 0)=v(\cdot, L)=0 & \text { in }(0, T) \\ u(0, \cdot)=0 & \text { in }(0, L)\end{cases}
$$


Using the fact that $\widehat{y}^{\alpha} \in L^{2}\left(0, T ; H^{2}(0, L)\right) \hookrightarrow L^{2}\left(0, T ; C^{1}[0, L]\right)$ and multiplying the first equation of the system above by $u$, we get:

$$
\begin{aligned}
\frac{1}{2} \frac{\mathrm{d}}{\mathrm{d} t}\|u\|_{2}^{2}+\left\|u_{x}\right\|_{2}^{2} & \leq\left\|z^{\alpha}\right\|_{\infty}\left\|u_{x}\right\|_{2}\|u\|_{2}+\left\|\widehat{y}_{x}^{\alpha}\right\|_{\infty}\|v\|_{2}\|u\|_{2} \\
& \leq \frac{1}{2}\left\|u_{x}\right\|_{2}^{2}+\frac{\left\|z^{\alpha}\right\|_{\infty}^{2}}{2}\|u\|_{2}^{2}+\left\|\widehat{y}_{x}^{\alpha}\right\|_{\infty}\|u\|_{2}^{2}
\end{aligned}
$$

Therefore,

$$
\frac{\mathrm{d}}{\mathrm{d} t}\|u\|_{2}^{2}+\left\|u_{x}\right\|_{2}^{2} \leq\left(\left\|z^{\alpha}\right\|_{\infty}^{2}+2\left\|\widehat{y}_{x}^{\alpha}\right\|_{\infty}\right)\|u\|_{2}^{2}
$$

Since $u(0, \cdot)=0$, Gronwall's Lemma implies $u \equiv 0$ and, consequently, $v \equiv 0$.

Finally, let us check that $z^{\alpha}$ satisfies the regularity properties in (2.5). To get this, let us introduce the function given by

$$
h(t, x):=\frac{v_{l}(t)(L-x)+x v_{r}(t)}{L} .
$$

Then, we obtain from (2.4) that $z^{\alpha}=w^{\alpha}+h$, where $w^{\alpha}$ solves

$$
\left\{\begin{array}{lll}
w^{\alpha}-\alpha^{2} w_{x x}^{\alpha}=y^{\alpha}-h \quad \text { in } & (0, T) \times(0, L) \\
w^{\alpha}(\cdot, 0)=w^{\alpha}(\cdot, 0)=0 & \text { in } \quad(0, T)
\end{array}\right.
$$

Consequently, $w^{\alpha} \in L^{2}\left(0, T ; H^{4}(0, L) \cap H_{0}^{1}(0, L)\right) \cap C^{0}\left([0, T] ; H^{3}(0, L) \cap H_{0}^{1}(0, L)\right)$ and the estimates are uniform, with respect to $\alpha$, in the space $L^{2}\left(0, T ; H^{2}(0, L) \cap H_{0}^{1}(0, L)\right) \cap C^{0}\left([0, T] ; H_{0}^{1}(0, L)\right)$.

Now, let us present a result concerning global existence and uniqueness of a (weak) solution with initial conditions in $L^{\infty}(0, L)$ :

Proposition 2.5. Let $\alpha>0, f \in L^{\infty}\left(0, T ; L^{\infty}(0, L)\right), y_{0} \in L^{\infty}(0, L)$ and $v_{l}, v_{r} \in H^{3 / 4}(0, T)$ be given. Then, there exists a unique solution $\left(y^{\alpha}, z^{\alpha}\right)$ to the Burgers- $\alpha$ system:

$$
\left\{\begin{array}{lll}
y_{t}^{\alpha}-y_{x x}^{\alpha}+z^{\alpha} y_{x}^{\alpha}=f & \text { in } & (0, T) \times(0, L), \\
z^{\alpha}-\alpha^{2} z_{x x}^{\alpha}=y^{\alpha} & \text { in } & (0, T) \times(0, L), \\
z^{\alpha}(\cdot, 0)=y^{\alpha}(\cdot, 0)=v_{l} & \text { in } & (0, T), \\
z^{\alpha}(\cdot, L)=y^{\alpha}(\cdot, L)=v_{r} & \text { in } & (0, T), \\
y^{\alpha}(0, \cdot)=y_{0} & \text { in } & (0, L)
\end{array}\right.
$$

with

$$
\left\{\begin{array}{l}
y^{\alpha} \in H^{1}\left(0, T ; H^{-1}(0, L)\right) \cap L^{2}\left(0, T ; H_{0}^{1}(0, L)\right) \cap C^{0}\left([0, T] ; L^{2}(0, L)\right) \cap L^{\infty}\left(0, T ; L^{\infty}(0, L)\right) \\
z^{\alpha} \in H^{1}\left(0, T ; H^{-1}(0, L)\right) \cap L^{2}\left(0, T ; H^{3}(0, L)\right) \cap C^{0}\left([0, T] ; H^{2}(0, L)\right)
\end{array}\right.
$$


Let us set $M_{T}:=\left\|y^{0}\right\|_{\infty}+\left\|v_{l}\right\|_{\infty}+\left\|v_{r}\right\|_{\infty}+T\|f\|_{\infty}$. Then, the following estimates holds:

$$
\begin{aligned}
\left\|y^{\alpha}\right\|_{\infty} & \leq M_{T}, \quad\left\|z^{\alpha}\right\|_{\infty} \leq M_{T} \\
\left\|y^{\alpha}\right\|_{H^{1}\left(H^{-1}\right) \cap L^{2}\left(H^{1}\right)}+\left\|y^{\alpha}\right\|_{L^{\infty}\left(L^{2}\right)} & \leq C e^{C M_{T}^{2}}\left(\|f\|_{2}+\left\|y_{0}\right\|_{2}+\left\|v_{l}\right\|_{H^{3 / 4}}+\left\|v_{r}\right\|_{H^{3 / 4}}\right), \\
\left\|z^{\alpha}\right\|_{2}+\alpha\left\|z_{x}^{\alpha}\right\|_{2}+\alpha^{2}\left\|z_{x x}^{\alpha}\right\|_{2} & \leq C e^{C M_{T}^{2}}\left[\|f\|_{2}+\left\|y_{0}\right\|_{2}+\left(1+\alpha^{2}\right)\left(\left\|\left(v_{l}, v_{r}\right)\right\|_{H^{3 / 4} \times H^{3 / 4}}\right)\right],
\end{aligned}
$$

where the constant $C>0$ is independent of $\alpha$.

Proof. Let $\xi \in L^{2}\left(0, T ; H^{2}(0, L)\right) \cap H^{1}\left(0, T ; L^{2}(0, L)\right)$ be such that

$$
\xi(\cdot, 0)=v_{l} \text { and } \xi(\cdot, L)=v_{r} \quad \text { in } \quad(0, T) .
$$

Now, for any $\bar{y} \in L^{2}\left(0, T ; L^{\infty}(0, L)\right)$, there exists a unique solution to the elliptic problem

$$
\begin{cases}z-\alpha^{2} z_{x x}=\bar{y} & \text { in }(0, T) \times(0, L) \\ z(\cdot, 0)=v_{l}, z(\cdot, L)=v_{r} & \text { in } \quad(0, T)\end{cases}
$$

and, again, using a lifting argument together with standard energy estimates for elliptic equtions, we get a positive constant $C$ (independent of $\alpha$ ) such that:

$$
\begin{aligned}
& \|z\|_{2}^{2}+2 \alpha^{2}\left\|z_{x}\right\|_{2}^{2}+\alpha^{4}\left\|z_{x x}\right\|_{2}^{2} \leq C\left(\|\bar{y}\|_{2}^{2}+\|\xi\|_{2}^{2}+\alpha^{2}\left\|\xi_{x}\right\|_{2}^{2}+\alpha^{4}\left\|\xi_{x x}\right\|_{2}^{2}\right) \\
& \|z\|_{L^{2}\left(L^{\infty}\right)} \leq\|\bar{y}\|_{L^{2}\left(L^{\infty}\right)}+\left\|v_{l}\right\|_{2}+\left\|v_{r}\right\|_{2}
\end{aligned}
$$

With this $z$, we can solve (for instance via Faedo-Galerkin method) the linear problem

$$
\begin{cases}y_{t}-y_{x x}+z y_{x}=f & \text { in } \quad(0, T) \times(0, L) \\ y(\cdot, 0)=v_{l}, y(\cdot, L)=v_{r} & \text { in }(0, T) \\ y(0, \cdot)=y_{0} & \text { in } \quad(0, L)\end{cases}
$$

and we find a weak solution $y$ that satisfies

$$
\left\|y_{t}\right\|_{L^{2}\left(H^{-1}\right)}+\|y\|_{L^{2}\left(H_{0}^{1}\right)}+\|y\|_{L^{\infty}\left(L^{2}\right)} \leq C\left(\left\|y_{0}\right\|_{2}+\|f\|_{2}+\left\|v_{l}\right\|_{H^{3 / 4}}+\left\|v_{r}\right\|_{H^{3 / 4}}\right) e^{C\|z\|_{L^{2}\left(L^{\infty}\right)}^{2}} .
$$

Again, as in the proof of Lemma 1 in [1], we can deduce that the solution to (2.13) satisfies

$$
\|y\|_{L^{\infty}\left(L^{\infty}\right)} \leq M_{T} \quad \text { and } \quad\|y\|_{L^{2}\left(L^{\infty}\right)} \leq T^{1 / 2} M_{T}
$$

Let us introduce the set

$$
K:=\left\{\bar{y} \in L^{2}\left(0, T ; L^{\infty}(0, L)\right):\|\bar{y}\|_{L^{2}\left(L^{\infty}\right)} \leq T^{1 / 2} M_{T}\right\}
$$

and the mapping $\Lambda_{\alpha}: K \mapsto K$ with $\Lambda_{\alpha}(\bar{y})=y$. Then, arguing as in the proof of Proposition 2.4, it is not difficult to prove that $\Lambda_{\alpha}$ possesses a fixed-point in $K$.

Finally, in order to prove uniqueness, we consider to solutions $u:=y^{\alpha}-\widehat{y}^{\alpha}$ and $v:=z^{\alpha}-\widehat{z}^{\alpha}$ and we get (2.9). Then, multiplying the first equation of (2.9) by $u$, we easily get the differential inequality

$$
\frac{\mathrm{d}}{\mathrm{d} t}\|u(t, \cdot)\|_{2}^{2}+\left\|u_{x}(t, \cdot)\right\|_{2}^{2} \leq\left(\left\|z^{\alpha}(t, \cdot)\right\|_{\infty}^{2}+\frac{2 C\left\|\widehat{y}_{x}^{\alpha}(t, \cdot)\right\|_{2}}{\alpha}\right)\|u(t, \cdot)\|_{2}^{2}
$$


Since $u(0, \cdot) \equiv 0$, Gronwall's Lemma implies $u \equiv 0$ and, consequently, $v \equiv 0$.

Let $\left(y^{\alpha}, z^{\alpha}\right)$ be the solution to (2.10). From (2.10) and the fact that $y \in L^{\infty}\left(0, T ; L^{\infty}(0, L)\right)$, the maximum principle implies that

$$
\left\|z^{\alpha}\right\|_{L^{\infty}\left(L^{\infty}\right)} \leq\left\|y^{\alpha}\right\|_{L^{\infty}\left(L^{\infty}\right)} \leq M_{T}
$$

This ends the proof.

\section{Controllability of the nON-Viscous Burgers- $\alpha$ System}

In this section we present a proof of the global exact controllability property of the non-viscous Burgers- $\alpha$ system. We split the proof in two parts: $(i)$ a local null controllability result; (ii) an argument based on a time scale-invariance and reversibility in time that leads to the desired global result.

\subsection{Local null controllability}

We have the following result:

Proposition 3.1. Let $\alpha>0$ be given. Then, there exist $\delta>0$ and $C>0$ (both independent of $\alpha$ ) such that the following property holds: for each $y_{0} \in C^{1}([0, L])$ with $\left\|y_{0}\right\|_{C^{1}([0, L])} \leq \delta$, there exist $p^{\alpha} \in C^{0}([0, T])$ with $p^{\alpha}(T)=0, v_{l}^{\alpha}, v_{r}^{\alpha} \in C^{1}([0, T])$ and associated states $\left(y^{\alpha}, z^{\alpha}\right) \in C^{1}\left([0, T] \times[0, L] ; \mathbb{R}^{2}\right)$ satisfying $(1.1)$,

$$
y^{\alpha}(T, \cdot)=z^{\alpha}(T, \cdot)=0 \quad \text { in } \quad(0, L)
$$

and

$$
\left\|p^{\alpha}\right\|_{C^{0}([0, T])}+\left\|\left(v_{l}^{\alpha}, v_{r}^{\alpha}\right)\right\|_{C^{1}\left([0, T] ; \mathbb{R}^{2}\right)} \leq C \quad \forall \alpha>0 .
$$

The proof is obtained by applying the return method, see $[11,15,16,32]$. It relies on a linearization process in combination with a fixed-point argument: $(i)$ first, we need to find a "good" trajectory (a particular solution for the nonlinear system) steering 0 to 0 such that the linearization around it is controllable; ( $i i)$ then, we must recover (for instance by a fixed-point argument) the exact controllability result, at least locally, for the nonlinear system.

In our case, it is not difficult to verify that the linearization around zero is not controllable. Accordingly, we build an appropriate nontrivial trajectory connecting $(0,0)$ to $(0,0)$.

To this purpose, let us introduce the set

$$
\Lambda_{L, T, k}:=\left\{\lambda \in C^{k}([0, T] ;[0, \infty)):\|\lambda\|_{L^{1}(0, T)}>L\right\} .
$$

Let us consider the couple $(\widehat{y}(x, t), \widehat{z}(x, t)):=(\lambda(t), \lambda(t))$ and the triplet $\left(\widehat{p}(t), \widehat{v}_{l}(t), \widehat{v}_{r}(t)\right):=\left(\lambda^{\prime}(t), \lambda(t), \lambda(t)\right)$, with $\lambda \in \Lambda_{L, T, 1}$ and supp $\lambda \subset(0, T)$. Note that $(\widehat{y}, \widehat{z})$ is a particular solution to (1.1), associated to the control $\left(\widehat{p}, \widehat{v}_{l}, \widehat{v}_{r}\right)$. We have the following general controllability result:

Proposition 3.2. Assume that $\lambda \in \Lambda_{L, T, 0}$ and let $\alpha>0$ be given. Then, for any $y_{0} \in C^{1}([0, L])$, there exists $(y, z) \in C^{1}\left([0, T] \times[0, L] ; \mathbb{R}^{2}\right)$ such that

$$
\left\{\begin{array}{lll}
y_{t}+\lambda(t) y_{x}=0 & \text { in }(0, T) \times(0, L), \\
z-\alpha^{2} z_{x x}=y & \text { in }(0, T) \times(0, L), \\
z(\cdot, 0)=y(\cdot, 0), \quad z(\cdot, L)=y(\cdot, L) & \text { in }(0, T), \\
y(0, \cdot)=y_{0} & \text { in }(0, L), \\
y(T, \cdot)=0 & \text { in }(0, L) .
\end{array}\right.
$$


For the proof, it suffices to use Proposition 8 of [11] to find $y \in C^{1}([0, T] \times[0, L])$ satisfying $(3.1)_{1},(3.1)_{4}$ and $(3.1)_{5}$ and then solve the elliptic problem $(3.1)_{2}-(3.1)_{3}$ to construct $z \in C^{1}([0, T] \times[0, L])$.

Thanks to Proposition 3.2, one may expect that the null controllability for the nonlinear system (1.1) holds. Indeed, we have the following result from which Proposition 3.1 is an immediate consequence:

Proposition 3.3. Assume that $\lambda \in \Lambda_{L, T, 0}$ and let $\alpha>0$ be given. Then, there exist $\delta>0$ and $C>0$ (both independent of $\alpha$ ) such that: for any $y_{0} \in C^{1}([0, L])$ with $\left\|y_{0}\right\|_{C^{1}([0, L])} \leq \delta$, there exist $\left(v_{l}, v_{r}\right) \in C^{1}\left([0, T] ; \mathbb{R}^{2}\right)$ and an associated state $(y, z) \in C^{1}\left([0, T] \times[0, L] ; \mathbb{R}^{2}\right)$ satisfying

$$
\begin{cases}y_{t}+(\lambda(t)+z) y_{x}=0 & \text { in }(0, T) \times(0, L), \\ z-\alpha^{2} z_{x x}=y & \text { in }(0, T) \times(0, L), \\ z(\cdot, 0)=v_{l}, \quad z(\cdot, L)=v_{r} & \text { in }[0, T] \\ y(\cdot, 0)=v_{l} & \text { in } I_{l} \\ y(\cdot, L)=v_{r} & \text { in } I_{r} \\ y(0, \cdot)=y_{0} & \text { in }(0, L), \\ y(T, \cdot)=0 & \text { in }(0, L)\end{cases}
$$

and

$$
\|y\|_{C^{1}([0, T] \times[0, L])} \leq C\left\|y_{0}\right\|_{C^{1}([0, L])} \quad \forall \alpha>0 .
$$

Proof. We will reformulate the null controllability problem as a fixed-point equation. To do this, we will first introduce some auxiliar functions and establish some helpful results. Thus, to any given $h \in$ $C^{0}\left([0, T] ; C^{0}([0, L])\right) \cap L^{\infty}\left(0, T ; C^{0,1}([0, L])\right)$, we associate the unique solution $z \in C^{0}\left([0, T] ; C^{2}([0, L])\right) \cap$ $L^{\infty}\left(0, T ; C^{3,1}([0, L])\right)$ to the time-dependent problem

$$
\left\{\begin{array}{lll}
z-\alpha^{2} z_{x x}=h \quad \text { in } & (0, T) \times(0, L), \\
z(\cdot, 0)=h(\cdot, 0) & \text { in } \quad(0, T), \\
z(\cdot, L)=h(\cdot, L) \quad \text { in } & (0, T) .
\end{array}\right.
$$

It follows from the maximum principle for elliptic equations that

$$
\|z(t, \cdot)\|_{\infty} \leq\|h(t, \cdot)\|_{\infty} \leq\|h\|_{C^{0}\left([0, T] ; C^{0}([0, L])\right)} \text { for } t \text { a.e. in }[0, T] .
$$

Since $h \in L^{\infty}\left(0, T ; C^{0,1}([0, L])\right)$, one has $h(t, \cdot) \in W^{1, \infty}(0, L)$ for $t$ a.e. in $[0, T]$ and, by applying again the maximum principle for elliptic equations, we get:

$$
\left\|z_{x}(t, \cdot)\right\|_{\infty} \leq\left\|h_{x}(t, \cdot)\right\|_{\infty} \leq\|h\|_{L^{\infty}\left(0, T ; C^{0,1}([0, L])\right)} \text { for } t \text { a.e. in }[0, T] .
$$

Then, by combining (3.4) and (3.5), we easily get that

$$
\|z\|_{C^{0}\left([0, T] ; C^{1}([0, L])\right)} \leq\|h\|_{C^{0}\left([0, T] ; C^{0}([0, L])\right) \cap L^{\infty}\left(0, T ; C^{0,1}([0, L])\right)}
$$

Note that this inequality does not lead to contradiction when alpha goes to 0 , since the space $C^{0}\left([0, T] ; C^{1}([0, L])\right)$ is not reflexive and, the limit, $z$ can "escape".

Since $\lambda \in \Lambda_{L, T, 0}$, we can find $\eta \in(0, L / 2)$ such that

$$
\int_{0}^{T} \lambda(s) \mathrm{d} s>L+2 \eta
$$


Now, we consider the following extension of $z$ to the closed interval $[-\eta, L+\eta]$ :

$$
z^{\eta}(t, x):= \begin{cases}5 z(t,-x)-20 z\left(t,-\frac{x}{2}\right)+16 z\left(t,-\frac{x}{4}\right), & (t, x) \in[0, T] \times[-\eta, 0], \\ z(t, x) & (t, x), \in[0, T] \times[0, L], \\ 5 z(t, 2 L-x)-20 z\left(t, \frac{3 L-x}{2}\right)+16 z\left(t, \frac{5 L-x}{4}\right), & (t, x) \in[0, T] \times[L, L+\eta] .\end{cases}
$$

It is not difficult to check that $z^{\eta} \in C^{0}\left([0, T] ; C^{2}([-\eta, L+\eta])\right)$ and there exists $C_{1}>0$ (independent of $\alpha$ ) such that

$$
\left\|z^{\eta}\right\|_{C^{0}\left([0, T] ; C^{1}([-\eta, L+\eta])\right)} \leq C_{1}\|z\|_{C^{0}\left([0, T] ; C^{1}([0, L])\right)} .
$$

Then, let $\chi$ be given, with $\chi \in C_{0}^{\infty}(-\eta / 2, L+\eta / 2), \chi=1$ in $[0, L]$ and $0 \leq \chi \leq 1$. This way, we can introduce $z^{*} \in C^{0}\left([0, T] ; C^{2}(\mathbb{R})\right)$, with

$$
z^{*}(t, x)= \begin{cases}\chi(x) z^{\eta}(t, x), & (t, x) \in[0, T] \times[-\eta, L+\eta] \\ 0, & (t, x) \in[0, T] \times(\mathbb{R} \backslash[-\eta, L+\eta]) .\end{cases}
$$

and, using (3.8) and (3.9), we see that

$$
\left\|z^{*}\right\|_{C^{0}\left([0, T] ; C_{b}^{1}(\mathbb{R})\right)} \leq C_{2}\|z\|_{C^{0}\left([0, T] ; C^{1}([0, L])\right)},
$$

for some $C_{2}>0$, again independent of $\alpha$.

Let us set $R:=\frac{\eta}{C_{2} T}$ and let us assume from now on that

$$
\|h\|_{C^{0}\left([0, T] ; C^{0}([0, L])\right) \cap L^{\infty}\left(0, T ; C^{0,1}([0, L])\right)} \leq R .
$$

Then, it follows from (3.6), (3.10) and (3.11) that

$$
\left\|z^{*}\right\|_{C^{0}\left([0, T] ; C_{b}^{1}(\mathbb{R})\right)} \leq \frac{\eta}{T} .
$$

Let $\phi^{*}$ be the flux associated to $\lambda+z^{*}$, that is, the solution to

$$
\left\{\begin{array}{l}
\frac{\partial \phi^{*}}{\partial t}(s ; t, x)=\lambda(t)+z^{*}\left(t, \phi^{*}(s ; t, x)\right) \\
\phi^{*}(s ; s, x)=x
\end{array}\right.
$$

Claim 3.4. The flux $\phi^{*}=\phi^{*}(s ; t, x)$ is well-defined for any $(t, x) \in[0, T] \times \mathbb{R}$ and $s \in[0, T]$.

Proof. Let $\phi:[0, T] \times[0, T] \times \mathbb{R} \mapsto \mathbb{R}$ be the flux associated to $\lambda$. Then, for every $(s, t, x) \in \operatorname{Dom}(\Phi)$, we get from (3.13) that

$$
\begin{aligned}
\left|\phi^{*}(s ; t, x)-\phi(s ; t, x)\right| & =\left|\int_{s}^{t}\left(\frac{\partial \phi^{*}}{\partial \tau}(s ; \tau, x)-\frac{\partial \phi}{\partial \tau}(s ; \tau, x)\right) \mathrm{d} \tau\right| \\
& \leq \int_{s}^{t}\left|\frac{\partial \phi^{*}}{\partial \tau}(s ; \tau, x)-\frac{\partial \phi}{\partial \tau}(s ; \tau, x)\right| \mathrm{d} \tau \\
& =\int_{s}^{t}\left|z^{*}\left(\tau, \phi^{*}(s ; \tau, x)\right)\right| \mathrm{d} \tau \\
& \leq T\left\|z^{*}\right\|_{C^{0}\left([0, T] ; C^{0}(\mathbb{R})\right) .}
\end{aligned}
$$


Hence, for any $(s, t, x)$ such that $\phi^{*}(s ; t, x)$ is well-defined, one has

$$
\left|\phi^{*}(s ; t, x)-\phi(s ; t, x)\right| \leq \eta .
$$

This and the fact that $\phi(s ; t, x)$ is well-defined for all $(s, t, x) \in[0, T] \times[0, L] \times \mathbb{R}$ lead to the desired conclusion.

Let $y_{0} \in C^{1}([0, L])$ be given and let us introduce $y_{0}^{\eta} \in C^{1}([-\eta, L+\eta])$ with

$$
y_{0}^{\eta}(x)= \begin{cases}-y^{0}(-x)+2 y^{0}(0), & x \in[-\eta, 0], \\ y^{0}(x), & x \in[0, L], \\ -y^{0}(2 L-x)+2 y^{0}(L), & x \in[L, L+\eta]\end{cases}
$$

and

$$
y_{0}^{*}(x)= \begin{cases}\chi(x) y_{0}^{\eta}(x), & x \in[-\eta, L+\eta], \\ 0, & x \in \mathbb{R} \backslash[-\eta, L+\eta] .\end{cases}
$$

Then, it is easy to see that $y_{0}^{*}$ is a compactly supported extension of $y_{0}$ to the whole real line and

$$
\left\|y_{0}^{*}\right\|_{C_{b}^{1}(\mathbb{R})} \leq C_{3}\left\|y_{0}\right\|_{C^{1}([0, L])}
$$

for some $C_{3}>0$ independent of $\alpha$.

Let us set $y \in C^{1}([0, T] \times \mathbb{R})$, with

$$
y(t, \bar{x}):=y_{0}^{*}\left(\phi^{*}(t ; 0, \bar{x})\right) \quad \forall(t, \bar{x}) \in[0, T] \times \mathbb{R} .
$$

Then, we have the following :

Claim 3.5. The function y satisfies:

$$
\begin{cases}y_{t}+\left(\lambda(t)+z^{*}(t, x)\right) y_{x}=0 & \text { in }(0, T) \times \mathbb{R}, \\ y(0, \cdot)=y_{0}^{*} & \text { in } \mathbb{R}, \\ y(T, \cdot)=0 & \text { in }[0, L] .\end{cases}
$$

Proof. For any $t \in[0, T], \phi^{*}(0 ; t, \cdot): \mathbb{R} \rightarrow \mathbb{R}$ is a diffeomorphism and $(3.16)$ is equivalent to $\left.y\left(t, \phi^{*}(0 ; t, x)\right)\right) \equiv$ $y_{0}^{*}(x)$. Then, for each $x \in \mathbb{R}$, we deduce that

$$
y_{t}\left(t, \phi^{*}(0 ; t, x)\right)+\left[\lambda(t)+z^{*}\left(t, \phi^{*}(0 ; t, x)\right)\right] y_{x}\left(t, \phi^{*}(0 ; t, x)\right)=0 .
$$

Using (3.16) and $(3.13)_{2}$, we get

$$
y(0, x)=y_{0}^{*}(x) \quad \forall x \in \mathbb{R} .
$$

Moreover, it is not difficult to see that, for any $0<\eta<L / 2$ such that (3.7) holds, the flux associated to $\lambda$ satisfies $\phi(T ; 0, L)<-2 \eta$ and we obtain from (3.14) that $\phi^{*}(T ; 0, L)<-\eta$. Since $\phi^{*}(s ; t, \cdot)$ is increasing for any 
$s, t \in[0, T]$, we see that

$$
\phi^{*}(T ; 0, x)<-\eta \quad \forall x \in(-\infty, L] .
$$

This inequality and (3.16) together with the fact that

$$
\operatorname{supp} y_{0}^{*} \subset[-\eta, L+\eta]
$$

imply that $y(T, \cdot)=0$ in $[0, L]$.

An immediate consequence of (3.12), (3.13), (3.15) and (3.16) is that

$$
\|y\|_{C^{1}([0, T] \times \mathbb{R})} \leq C_{4}\left\|y_{0}\right\|_{C^{1}([0, L])}
$$

for a positive constant $C_{4}$ depending on $R$ but independent of $\alpha$. By taking $y_{0} \in C^{1}([0, L])$ such that

$$
\left\|y_{0}\right\|_{C^{1}([0, L])} \leq R / 3 C_{4},
$$

we get easily the function $y$, defined in (3.16), satisfies the inequality

$$
\|y\|_{C^{0}\left([0, T] ; C^{0}(\mathbb{R})\right)}+\|y\|_{L^{\infty}\left(0, T ; C^{0,1}(\mathbb{R})\right)} \leq R .
$$

Let us consider now the closed ball $B_{R}$ centered at the origin and radius $R$ in the Banach space $C^{0}\left([0, T] ; C^{0}([0, L])\right) \cap L^{\infty}\left(0, T ; C^{0,1}([0, L])\right)$. Using the inequality (3.18), we can define the mapping $\mathcal{F}: B_{R} \mapsto$ $B_{R}$, with $\mathcal{F}(h)=\left.y\right|_{[0, T] \times[0, L]}$, where $y$ is given by $(3.16)$.

Thanks to $(3.16)$, we have that $\mathcal{F}\left(B_{R}\right) \subset C^{1}([0, T] \times[0, L])$. Furthermore, the following holds:

Claim 3.6. There exists a positive constant $C_{5}$ depending on $\left\|y_{0}\right\|_{C^{1}([0, L])}, L, R$ and $T$, but independent of $\alpha$, such that, for any $m \geq 1$ and any $h^{1}, h^{2} \in B_{R}$, one has

$$
\left\|\left(\mathcal{F}^{m}\left(h^{1}\right)-\mathcal{F}^{m}\left(h^{2}\right)\right)(t, \cdot)\right\|_{C^{0}([0, L])} \leq \frac{\left(C_{5} t\right)^{m}}{m !}\left\|h^{1}-h^{2}\right\|_{C^{0}\left([0, T] ; C^{0}([0, L])\right)} \text { in }[0, T],
$$

where $\mathcal{F}^{m}$ denotes $\mathcal{F}$ iterated $m$ times.

Proof. The proof relies on an induction argument. Let $h^{i} \in B_{R}$ be given for $i=1,2$. Then, let us consider the functions $z^{i, *}$ and $y^{i}$, respectively given by (3.9) and (3.16) and set $y:=y^{1}-y^{2}$ and $z^{*}:=z^{1, *}-z^{2, *}$. By Claim 3.5, we have

$$
y_{t}+\left(\lambda+z^{1, *}\right) y_{x}=-z^{*} y_{x}^{2} \quad \text { in } \quad(0, T) \times \mathbb{R}
$$

whence, from Proposition 2.2,

$$
\frac{\mathrm{d}}{\mathrm{d} t^{+}}\|y(t, \cdot)\|_{C_{b}^{0}(\mathbb{R})} \leq\left\|z^{*}(t, \cdot) y_{x}^{2}(t, \cdot)\right\|_{C_{b}^{0}(\mathbb{R})} .
$$

Therefore, integrating from 0 to $t$ and using that $y_{x}^{2} \in C^{0}\left([0, T] ; C_{b}^{0}(\mathbb{R})\right)$ and the maximum principle for elliptic PDE's, we find a positive constant $C_{5}$ depending on $\left\|y_{0}\right\|_{C^{1}([0, L])}, L, R$ and $T$, but independent of $\alpha$, such that

$$
\|y(t, \cdot)\|_{C_{b}^{0}(\mathbb{R})} \leq R \int_{0}^{t}\left\|z^{*}(\tau, \cdot)\right\|_{C_{b}^{0}(\mathbb{R})} \mathrm{d} \tau
$$




$$
\begin{aligned}
& \leq C_{5} \int_{0}^{t}\left\|z^{1}(\tau, \cdot)-z^{2}(\tau, \cdot)\right\|_{C^{0}([0, L])} \mathrm{d} \tau \\
& \leq C_{5} \int_{0}^{t}\left\|h^{1}(\tau, \cdot)-h^{2}(\tau, \cdot)\right\|_{C^{0}([0, L])} \mathrm{d} \tau .
\end{aligned}
$$

It follows that

$$
\left\|\left(\mathcal{F}\left(h^{1}\right)-\mathcal{F}\left(h^{2}\right)\right)(t, \cdot)\right\|_{C^{0}([0, L])} \leq C_{5} t\left\|h^{1}-h^{2}\right\|_{C^{0}\left([0, T] ; C^{0}([0, L])\right)}
$$

and the result is true for $m=1$.

Then, by making similar computations and using the induction hypothesis, we get the result of the claim.

Let $\widetilde{B}_{R}$ be the closure of $B_{R}$ with the norm of $C^{0}\left([0, T] ; C^{0}([0, L])\right)$ and let $\widetilde{\mathcal{F}}$ be the unique continuous extension of $\mathcal{F}$ to $\widetilde{B}_{R}$. Note that, since $\mathcal{F}$ is uniformly continuous, $\widetilde{\mathcal{F}}$ is well-defined.

Claim 3.7. The continuous extension $\widetilde{\mathcal{F}}$ satisfies the following properties:

a) For any $h$ in $\widetilde{B}_{R}$, the function $\widetilde{\mathcal{F}}(h)$ belongs to $C^{1}([0, T] \times[0, L])$ and satisfies equation (3.17);

b) $\widetilde{\mathcal{F}}\left(\widetilde{B}_{R}\right) \subset B_{R}$.

Proof. Let us begin by proving the item $a$ ). For a given $h \in \widetilde{B}_{R}$, let us consider a sequence $\left(h_{n}\right)_{n \in \mathbb{N}}$ in $B_{R}$ such that $h_{n} \rightarrow h$ in $C^{0}\left([0, T] ; C^{0}([0, L])\right)$. Therefore, the corresponding elliptic solutions to (3.3) and the associated flows (given in (3.13)) satisfy the convergences

$$
z_{n} \rightarrow z \text { in } C^{0}\left([0, T] ; C^{2}([0, L])\right) \text { and } \Phi_{n}^{*} \rightarrow \Phi^{*} \text { in } C^{0}([0, T] \times[0, T] \times \mathbb{R}) .
$$

Moreover, since the $\Phi_{n}^{*}, \Phi^{*} \in C^{1}([0, T] \times[0, T] \times \mathbb{R})$, the corresponding functions, defined in (3.16), belong to $C^{1}([0, T] \times[0, L])$, verify the transport equation (3.17) and, furthermore,

$$
y_{n} \rightarrow y \text { in } C^{0}\left([0, T] ; C^{0}([0, L])\right) .
$$

Thus, it follows easily from this and from the definition of $\mathcal{F}$ and $\widetilde{\mathcal{F}}$ that $\widetilde{\mathcal{F}}(h)=y$.

Let us now prove the item $b$ ). In fact, notice that, by definition of $\mathcal{F}$, for any $h \in \widetilde{B}_{R}$ there exists a sequence $\left(h_{n}\right)_{n=1}^{\infty}$ in $B_{R}$ such that $h_{n} \rightarrow h$ in $C^{0}\left([0, T] ; C^{0}([0, L])\right)$ and

$$
\widetilde{\mathcal{F}}(h)=\lim _{n \rightarrow \infty} \mathcal{F}\left(h_{n}\right) \text { in } C^{0}\left([0, T] ; C^{0}([0, L])\right) .
$$

It is not difficult to prove that

$$
\|\widetilde{\mathcal{F}}(h)(t, \cdot)\|_{C^{0}([0, L])} \leq\left\|\mathcal{F}\left(h_{n}\right)(t, \cdot)\right\|_{C^{0}([0, L])}+\left\|\mathcal{F}\left(h_{n}\right)(t, \cdot)-\widetilde{\mathcal{F}}(h)(t, \cdot)\right\|_{C^{0}([0, L])}, \quad \forall n \in \mathbb{N} .
$$

On the other hand, since $\widetilde{\mathcal{F}}(h), \mathcal{F}\left(h_{n}\right) \in C^{1}([0, T] \times[0, L])$, we have, for any $x, x^{\prime} \in[0, L]$ with $x \neq x^{\prime}$, that

$$
\begin{aligned}
& \frac{\left|\widetilde{\mathcal{F}}(h)(t, x)-\widetilde{\mathcal{F}}(h)\left(t, x^{\prime}\right)\right|}{\left|x-x^{\prime}\right|} \leq \frac{\left|\mathcal{F}\left(h_{n}\right)(t, x)-\mathcal{F}\left(h_{n}\right)\left(t, x^{\prime}\right)\right|}{\left|x-x^{\prime}\right|} \\
& \quad+\frac{\left|\left(\widetilde{\mathcal{F}}(h)(t, x)-\mathcal{F}\left(h_{n}\right)(t, x)\right)-\left(\widetilde{\mathcal{F}}(h)\left(t, x^{\prime}\right)-\mathcal{F}\left(h_{n}\right)\left(t, x^{\prime}\right)\right)\right|}{\left|x-x^{\prime}\right|}
\end{aligned}
$$


and, consequently, letting $n \rightarrow+\infty$ and then maximizing in $x$ and $x^{\prime}$, the following is found:

$$
\sup _{x, x^{\prime} \in[0, L]} \frac{\left|\widetilde{\mathcal{F}}(h)(t, x)-\widetilde{\mathcal{F}}(h)\left(t, x^{\prime}\right)\right|}{\left|x-x^{\prime}\right|} \leq \sup _{n \in \mathbb{N}}\left(\sup _{x, x^{\prime} \in[0, L]} \frac{\left|\mathcal{F}\left(h_{n}\right)(t, x)-\mathcal{F}\left(h_{n}\right)\left(t, x^{\prime}\right)\right|}{\left|x-x^{\prime}\right|}\right) .
$$

Therefore, using the fact that $\widetilde{\mathcal{F}}(h) \in C^{1}([0, T] \times[0, L])$ and the $\mathcal{F}\left(h_{n}\right)$ belong to $B_{R}$, we certainly have $\widetilde{\mathcal{F}}(h) \in B_{R}$.

It follows from Claim 3.6 that $\mathcal{F}^{m}$ is a contraction for $m$ large enough. Then, from the Theorem $2.3, \widetilde{\mathcal{F}}$ possesses a unique fixed-point $y \in \widetilde{B}_{R}$. Finally, taking into account Claim 3.7, the proof of Proposition 3.3 is achieved.

Remark 3.8. Assume that $\lambda \in \Lambda_{L, T, 1}$ and consider the Banach space

$$
\mathcal{X}=C^{0}\left([0, T] ; C^{1}([0, L])\right) \cap C^{1}\left([0, T] ; C^{0}([0, L])\right) \cap L^{\infty}\left(0, T ; C^{1,1}([0, L])\right)
$$

If $y_{0} \in C^{2}([0, L])$ is small enough, then the fixed-point mapping $\mathcal{F}$ can be defined in a closed ball of $\mathcal{X}$ centered at zero of radius $R>0$. Then, one applies the Theorem 2.3 to the closure of this ball with the norm of $C^{0}\left([0, T] ; C^{1}([0, L])\right) \cap C^{1}\left([0, T] ; C^{0}([0, L])\right)$. Performing similar computations as those in Proposition 3.3, one can deduce that there exists $\delta>0$ (independent of $\alpha$ ) such that, for any $y_{0} \in C^{2}([0, L])$ with $\left\|y_{0}\right\|_{C^{2}([0, L])} \leq \delta$, there exists a solution $(y, z) \in C^{2}\left([0, T] \times[0, L] ; \mathbb{R}^{2}\right)$ to $(3.2)$, satisfying

$$
\|y\|_{\left.C^{2}([0, T] \times[0, L])\right)} \leq C\left\|y_{0}\right\|_{C^{2}([0, L])} \quad \forall \alpha>0,
$$

for a constant $C>0$ that is independent of $\alpha$.

Remark 3.9. Proposition 3.1 is a consequence of Proposition 3.3 by assuming that $\lambda \in \Lambda_{L, T, 1}$ and satisfies $\lambda(0)=\lambda(T)=\lambda_{t}(T)=0$.

Remark 3.10. From the proof of the previous result, one sees that $z(\cdot, 0)=y(\cdot, 0)$ and $z(\cdot, L)=y(\cdot, L)$ in $[0, T]$. This is important at least for two reasons: $(i)$ to guarantee that in the limit, as $\alpha$ goes to $0, z$ and $y$ converge to the same limit; (ii) to build suitable controls for the uniform approximate controllability problem presented in Section 4.2.

\subsection{Global exact controllability}

In order to prove Theorem 1.1, we have to use scaling arguments and the time-reversibility of the nonviscous Burgers- $\alpha$ system. Thus, let us consider initial and final states $y_{0}, y_{T} \in C^{1}([0, L])$, let $\delta>0$ be given by Proposition 3.1 and let $\gamma_{0}, \gamma_{T} \in(0,1)$ be such that $\gamma_{0}<\gamma_{T}$,

$$
\left\|\gamma_{0} y_{0}\right\|_{C^{1}([0, L])} \leq \delta \text { and }\left\|\left(1-\gamma_{T}\right) y_{T}\right\|_{C^{1}([0, L])} \leq \delta
$$


Then, by Proposition 3.1, there exist distributed controls $\widetilde{p}, \widehat{p}$ in $C^{0}([0, T])$, with $\widetilde{p}(T)=\widehat{p}(T)=0$, boundary controls $\left(\widetilde{v}_{l}, \widetilde{v}_{r}\right),\left(\widehat{v}_{l}, \widehat{v}_{r}\right)$ in $C^{1}([0, T])$ and associated states $(\widetilde{y}, \widetilde{z}),(\widehat{y}, \widehat{z})$ in the space $C^{1}([0, T] \times[0, L])$ such that

$$
\begin{cases}\widetilde{y}_{t}+\widetilde{z} \widetilde{y}_{x}=\widetilde{p}(t) & \text { in } \quad(0, T) \times(0, L), \\ \widetilde{z}-\alpha^{2} \widetilde{z}_{x x}=\widetilde{y} & \text { in } \quad(0, T) \times(0, L), \\ \widetilde{z}(\cdot, 0)=\widetilde{v}_{l}, \widetilde{z}(\cdot, L)=\widetilde{v}_{r} & \text { in } \quad(0, T), \\ \widetilde{y}(\cdot, 0)=\widetilde{v}_{l} & \text { in } \widetilde{I}_{l}, \\ \widetilde{y}(\cdot, L)=\widetilde{v}_{r} & \text { in } \widetilde{I}_{r}, \\ \widetilde{y}(0, \cdot)=\gamma_{0} y_{0}(x) & \text { in }(0, L), \\ \widetilde{y}(T, \cdot)=0 & \text { in }(0, L),\end{cases}
$$

where $\widetilde{I}_{l}=\left\{t \in[0, T]: \widetilde{v}_{l}(t)>0\right\}$ and $\widetilde{I}_{r}=\left\{t \in[0, T]: \widetilde{v}_{r}(t)<0\right\}$ and

$$
\begin{cases}\widehat{y}_{t}+\widehat{z} \widehat{y}_{x}=\widehat{p}(t) & \text { in } \quad(0, T) \times(0, L), \\ \widehat{z}-\alpha \widehat{z}_{x x}=\widehat{y} & \text { in }[0, T] \times[0, L], \\ \widehat{z}(\cdot, 0)=\widehat{v}_{l}, \widehat{z}(\cdot, L)=\widehat{v}_{r} & \text { in }(0, T), \\ \widehat{y}(\cdot, 0)=\widehat{v}_{l} & \text { in } \widehat{I}_{l}, \\ \widehat{y}(\cdot, L)=\widehat{v}_{r} & \text { in } \widehat{I}_{r}, \\ \widehat{y}(0, \cdot)=\left(1-\gamma_{T}\right) y_{T} & \text { in }(0, L), \\ \widehat{y}(T, \cdot)=0 & \text { in }(0, L),\end{cases}
$$

where $\widehat{I}_{l}=\left\{t \in[0, T]: \widehat{v}_{l}(t)>0\right\}$ and $\widehat{I}_{r}=\left\{t \in[0, T]: \widehat{v}_{r}(t)<0\right\}$.

Using (3.21), (3.22) and the fact that $\widetilde{p}(T)=\widehat{p}(T)=0$ and $\gamma_{0} \sim 0$ and $\gamma_{T} \sim 1$, we can introduce the functions $Y, Z:[0, T] \times[0, L] \mapsto \mathbb{R}$ and $P, V_{l}, V_{r}:[0, T] \mapsto \mathbb{R}$, given by

$$
\begin{gathered}
Y(t, x):= \begin{cases}\gamma_{0}^{-1} \widetilde{y}\left(t \gamma_{0}^{-1}, x\right) & (t, x) \in\left[0, \gamma_{0} T\right] \times[0, L], \\
0 & (t, x) \in\left[\gamma_{0} T, \gamma_{T} T\right] \times[0, L], \\
\frac{1}{1-\gamma_{T}} \widehat{y}\left(\frac{T-t}{1-\gamma_{T}}, L-x\right) & (t, x) \in\left[\gamma_{T} T, T\right] \times[0, L],\end{cases} \\
Z(t, x):= \begin{cases}\gamma_{0}^{-1} \widetilde{z}\left(t \gamma_{0}^{-1}, x\right) & (t, x) \in\left[0, \gamma_{0} T\right] \times[0, L], \\
0 & (t, x) \in\left[\gamma_{0} T, \gamma_{T} T\right] \times[0, L], \\
\frac{1}{1-\gamma_{T}} \widehat{z}\left(\frac{T-t}{1-\gamma_{T}}, L-x\right) & (t, x) \in\left[\gamma_{T} T, T\right] \times[0, L],\end{cases} \\
P(t):= \begin{cases}\gamma_{0}^{-2} \widetilde{p}\left(t \gamma_{0}^{-1}\right) & t \in\left[0, \gamma_{0} T\right], \\
0 & t \in\left[\gamma_{0} T, \gamma_{T} T\right], \\
-\frac{1}{\left(1-\gamma_{T}\right)^{2}} \widehat{p}\left(\frac{T-t}{1-\gamma_{T}}\right) & t \in\left[\gamma_{T} T, T\right],\end{cases} \\
V_{l}(t):= \begin{cases}\gamma_{0}^{-1} \widetilde{v}_{l}\left(t \gamma_{0}^{-1}, x\right) & t \in\left[0, \gamma_{0} T\right], \\
0 & t \in\left[\gamma_{0} T, \gamma_{T} T\right], \\
\frac{1}{1-\gamma_{T}} \widehat{v}_{r}\left(\frac{T-t}{1-\gamma_{T}}\right) & t \in\left[\gamma_{T} T, T\right]\end{cases}
\end{gathered}
$$


and

$$
V_{r}(t):= \begin{cases}\gamma_{0}^{-1} \widetilde{v}_{r}\left(t \gamma_{0}^{-1}, x\right) & t \in\left[0, \gamma_{0} T\right], \\ 0 & t \in\left[\gamma_{0} T, \gamma_{T} T\right] \\ \frac{1}{1-\gamma_{T}} \widehat{v}_{l}\left(\frac{T-t}{1-\gamma_{T}}\right) & t \in\left[\gamma_{T} T, T\right] .\end{cases}
$$

It is now straightforward to check that $(Y, Z) \in C^{1}\left([0, T] \times[0, L] ; \mathbb{R}^{2}\right), P \in C^{0}([0, T]), V_{l}, V_{r} \in C^{1}([0, T])$ and (1.1), (1.4) and (1.5) are satisfied.

Remark 3.11. Notice that the distributed control $p^{\alpha}$ is independent of $\alpha$, that is, its definition only depends on $T, L$, the initial condition and the target state.

\section{Global controllability of the viscous Burgers- $\alpha$ System}

\subsection{Smoothing effect}

The goal of this section is to prove that, starting from a $H_{0}^{1}$ initial state, there exists a small time where the solution begins to be smooth. More precisely, we have the following result:

Proposition 4.1. Let $y_{0} \in H_{0}^{1}(0, L)$ be given and let $\left(y^{\alpha}, z^{\alpha}\right)$ be the solution to

$$
\left\{\begin{array}{lll}
y_{t}^{\alpha}-y_{x x}^{\alpha}+z^{\alpha} y_{x}^{\alpha}=0 & \text { in }(0, T) \times(0, L), \\
z^{\alpha}-\alpha^{2} z_{x x}^{\alpha}=y^{\alpha} & \text { in }(0, T) \times(0, L), \\
y^{\alpha}(\cdot, 0)=y^{\alpha}(\cdot, L)=z^{\alpha}(\cdot, 0)=z^{\alpha}(\cdot, L)=0 & \text { in }(0, T), \\
y^{\alpha}(0, \cdot)=y_{0} & \text { in }(0, L) .
\end{array}\right.
$$

Then, there exist $T^{*} \in(0, T / 2)$ such that the solution $y^{\alpha}$ belongs to $C^{0}\left(\left[T^{*}, T\right] ; H^{3}(0, L)\right)$ and satisfies

$$
\left\|y^{\alpha}\right\|_{C^{0}\left(\left[T^{*}, T\right] ; H^{3}(0, L)\right)} \leq \Lambda\left(\left\|y_{0}\right\|_{H_{0}^{1}}\right)
$$

where $\Lambda: \mathbb{R}_{+} \rightarrow \mathbb{R}_{+}$is a continuous function satisfying $\Lambda(s) \rightarrow 0$ as $s \rightarrow 0^{+}$.

Proof. We will divide the proof in several steps. Throughout the proof, all the constants are independent of $\alpha$.

Step 1: Strong estimates in $(0, T / 2)$. Since $y_{0} \in H_{0}^{1}(0, L), f \equiv 0$ and $v_{l} \equiv v_{r} \equiv 0$, Proposition 2.4 implies the existence and uniqueness of a solution $\left(y^{\alpha}, z^{\alpha}\right)$ to (4.1) satisfying (2.5) and (2.6). In particular, we have from (2.6) that

$$
\left\|y^{\alpha}\right\|_{L^{\infty}\left(H_{0}^{1}\right)}+\left\|y^{\alpha}\right\|_{L^{2}\left(H^{2} \cap H_{0}^{1}\right)} \leq C\left\|y_{0}\right\|_{H_{0}^{1}} e^{C\left\|y_{0}\right\|_{H_{0}^{1}}^{2}}
$$

Consequently, there exists $t_{1} \in(0, T / 2)$ such that

$$
\left\|y^{\alpha}\left(t_{1}, \cdot\right)\right\|_{H^{2} \cap H_{0}^{1}} \leq \sqrt{\frac{2}{T}} C\left\|y_{0}\right\|_{H_{0}^{1}} e^{C\left\|y_{0}\right\|_{H_{0}^{1}}^{2}}
$$


Step 2: Estimates in $\left(t_{1}, T / 2\right)$. Let us set $y_{1}:=y^{\alpha}\left(t_{1}, \cdot\right), g:=z^{\alpha} y_{x}^{\alpha}$. Then, we can easily check that $y^{\alpha}$ is the unique solution to the heat equation:

$$
\left\{\begin{array}{lll}
y_{t}^{\alpha}-y_{x x}^{\alpha}=g & \text { in } & \left(t_{1}, T\right) \times(0, L) \\
y^{\alpha}(\cdot, 0)=y^{\alpha}(\cdot, L)=0 & \text { in } & \left(t_{1}, T\right), \\
y^{\alpha}\left(t_{1}, \cdot\right)=y_{1} & \text { in } & (0, L) .
\end{array}\right.
$$

From the regularity of $y^{\alpha}$ and $z^{\alpha}$, we have $g \in L^{2}\left(0, T ; H_{0}^{1}(0, L)\right) \cap H^{1}\left(0, T ; H^{-1}(0, L)\right)$ and

$$
\begin{aligned}
\|g\|_{L^{2}\left(H_{0}^{1}\right)}+\left\|g_{t}\right\|_{L^{2}\left(H^{-1}\right)} & \leq C\left\|y^{\alpha}\right\|_{L^{\infty}\left(H_{0}^{1}\right)}\left(\left\|y^{\alpha}\right\|_{L^{2}\left(H^{2}\right)}+\left\|y_{t}^{\alpha}\right\|_{L^{2}\left(L^{2}\right)}\right) \\
& \leq C e^{C\left\|y_{0}\right\|_{H_{0}^{1}}^{2}\left\|y_{0}\right\|_{H_{0}^{1}}^{2}}
\end{aligned}
$$

Using this estimate, the fact that $g \in C^{0}\left([0, T] ; L^{2}(0, L)\right)$ (see [24], Chap. 5, Thm. 3), (4.2) and the parabolic regularity result ([24], Ch. 3, Thm. 5), we find that

$$
y^{\alpha} \in L^{\infty}\left(t_{1}, T ; H^{2}(0, L)\right), y_{t}^{\alpha} \in L^{\infty}\left(t_{1}, T ; L^{2}(0, L)\right) \cap L^{2}\left(t_{1}, T ; H_{0}^{1}(0, L)\right) \cap H^{1}\left(t_{1}, T ; H^{-1}(0, L)\right)
$$

and, in the time interval $\left(t_{1}, T\right)$,

$$
\begin{aligned}
\left\|y^{\alpha}\right\|_{L^{\infty}\left(H^{2}\right)}+\left\|y_{t}^{\alpha}\right\|_{L^{\infty}\left(L^{2}\right) \cap L^{2}\left(H_{0}^{1}\right)}+\left\|y_{t t}^{\alpha}\right\|_{L^{2}\left(H^{-1}\right)} & \leq C\left(\|g\|_{L^{2}\left(H_{0}^{1}\right) \cap H^{1}\left(H^{-1}\right)}+\left\|y_{1}\right\|_{H^{2}}\right) \\
& \leq \frac{1}{2} \Lambda_{1}\left(\left\|y_{0}\right\|_{H_{0}^{1}}\right)
\end{aligned}
$$

where

$$
\Lambda_{1}\left(\left\|y_{0}\right\|_{H_{0}^{1}}\right)=2 C e^{C\left\|y_{0}\right\|_{H_{0}^{1}}^{2}\left\|y_{0}\right\|_{H_{0}^{1}}\left(1+\left\|y_{0}\right\|_{H_{0}^{1}}\right)}
$$

From (4.2), we have that

$$
\left\{\begin{array}{l}
-y_{x x}^{\alpha}(t, \cdot)=g(t, \cdot)-y_{t}^{\alpha}(t, \cdot) \\
y^{\alpha}(t, 0)=y^{\alpha}(t, L)=0
\end{array}\right.
$$

for $t$ a.e in $\left(t_{1}, T\right)$. Thus, using (4.3) and elliptic regularity results, (see [24], Chap. 6, Thm. 5), we deduce that $y^{\alpha} \in L^{2}\left(t_{1}, T ; H^{3}(0, L)\right)$ and

$$
\left\|y^{\alpha}\right\|_{L^{2}\left(t_{1}, T ; H^{3}(0, L)\right)} \leq \frac{1}{2} \Lambda_{1}\left(\left\|y_{0}\right\|_{H_{0}^{1}}\right) .
$$

We also deduce that, for some $t_{2} \in\left(t_{1}, T / 2\right)$, one has

$$
\left\|y_{t}^{\alpha}\left(t_{2}, \cdot\right)\right\|_{H_{0}^{1}}+\left\|y^{\alpha}\left(t_{2}, \cdot\right)\right\|_{H^{3} \cap H_{0}^{1}} \leq \sqrt{\frac{2}{T-2 t_{1}}} \Lambda_{1}\left(\left\|y_{0}\right\|_{H_{0}^{1}}\right) .
$$

Step 3: Estimates in $\left(t_{2}, T / 2\right)$. Let us set $y_{2}:=y^{\alpha}\left(t_{2}, \cdot\right)$. Note that

$$
\begin{aligned}
\|g\|_{L^{2}\left(t_{1}, T ; H^{2}(0, L)\right) \cap H^{1}\left(t_{1}, T ; L^{2}(0, L)\right)} & \leq C\left\|y^{\alpha}\right\|_{L^{\infty}\left(t_{1}, T ; H_{0}^{1}(0, L)\right)}\left\|y^{\alpha}\right\|_{L^{2}\left(t_{1}, T ; H^{3}(0, L)\right) \cap H^{1}\left(t_{1}, T ; H_{0}^{1}(0, L)\right)} \\
& \leq C\left\|y_{0}\right\|_{H_{0}^{1}} \Lambda_{1}\left(\left\|y_{0}\right\|_{H_{0}^{1}}\right) e^{C\left\|y_{0}\right\|_{H_{0}^{1}}^{2}}
\end{aligned}
$$


and the needed compatibility conditions for regularity results holds:

$$
g\left(t_{2}, \cdot\right)+y_{2, x x}=y_{t}^{\alpha}\left(t_{2}, \cdot\right) \in H_{0}^{1}(0, L)
$$

Using ([24], Ch. 7, Thm. 6), we get that

$$
y^{\alpha} \in L^{2}\left(t_{2}, T ; H^{4}(0, L)\right) \cap H^{1}\left(t_{2}, T ; H^{2}(0, L)\right) \cap H^{2}\left(t_{2}, T ; L^{2}(0, L)\right)
$$

and, moreover, in the time interval $\left(t_{2}, T\right)$

$$
\begin{aligned}
\left\|y^{\alpha}\right\|_{L^{2}\left(H^{4}\right) \cap H^{1}\left(H^{2}\right) \cap H^{2}\left(L^{2}\right)} & \leq C\left(\|g\|_{L^{2}\left(H^{2}\right) \cap H^{1}\left(L^{2}\right)}+\left\|y_{2}\right\|_{H^{3}}\right) \\
& \leq \Lambda_{2}\left(\left\|y_{0}\right\|_{H_{0}^{1}}\right)
\end{aligned}
$$

where

$$
\Lambda_{2}\left(\left\|y_{0}\right\|_{H_{0}^{1}}\right):=C\left(1+\left\|y_{0}\right\|_{H_{0}^{1}} e^{C\left\|y_{0}\right\|_{H_{0}^{1}}^{2}}\right) \Lambda_{1}\left(\left\|y_{0}\right\|_{H_{0}^{1}}\right)
$$

Step 4: Conclusion. Finally, the result in ([24], Ch. 5, Thm. 4) applied to (4.4) leads to the regularity $C^{0}\left(\left[t_{2}, T\right] ; H^{3}(0, L)\right)$ for $y^{\alpha}$. Therefore, the conclusion follows from Sobolev's embedding, taking $T^{*}=t_{2}$ and $\Lambda\left(\left\|y_{0}\right\|_{H_{0}^{1}}\right)=\Lambda_{2}\left(\left\|y_{0}\right\|_{H_{0}^{1}}\right)$.

Remark 4.2. Proposition 4.1 is also true when $y_{0} \in L^{\infty}(0, L)$. Indeed, we can start using Proposition 2.4 that guarantees the existence and uniqueness of a solution $\left(y^{\alpha}, z^{\alpha}\right)$ to (4.1) satisfying (2.11) and (2.12). In particular, we have from (2.12) that

$$
\left\|y^{\alpha}\right\|_{L^{2}\left(H_{0}^{1}\right)} \leq C\left\|y_{0}\right\|_{\infty} e^{C\left\|y_{0}\right\|_{\infty}^{2}}
$$

Therefore, there exists $t_{1} \in(0, T / 2)$ such that

$$
\left\|y^{\alpha}\left(t_{1}, \cdot\right)\right\|_{H_{0}^{1}} \leq \sqrt{\frac{2}{T}} C\left\|y_{0}\right\|_{\infty} e^{C\left\|y_{0}\right\|_{\infty}^{2}}
$$

Then, we can achieve arguing as in the proof of Proposition 4.1.

\subsection{Uniform approximate controllability}

In this section, the goal is to prove the following approximate controllability result starting from sufficiently smooth initial data:

Proposition 4.3. Let $y_{0}, y_{f} \in C^{2}([0, L])$ be given. There exist positive constants $\tau_{*}$ and $K>0$, both independent of $\alpha$, such that, for any $\tau \in\left(0, \tau_{*}\right]$, there exist $p^{\alpha} \in C_{c}^{0}(0, \tau),\left(v_{l}^{\alpha}, v_{r}^{\alpha}\right) \in H^{3 / 4}\left(0, \tau ; \mathbb{R}^{2}\right)$ and associated states $\left(y^{\alpha}, z^{\alpha}\right)$ with the following regularity

$$
\left\{\begin{array}{l}
y^{\alpha} \in L^{2}\left(0, \tau ; H^{2}(0, L)\right) \cap H^{1}\left(0, \tau ; L^{2}(0, L)\right) \cap C^{0}\left([0, \tau] ; H^{1}(0, L)\right) \\
z^{\alpha} \in L^{2}\left(0, T ; H^{4}(0, L)\right) \cap H^{1}\left(0, \tau ; L^{2}(0, L)\right) \cap C^{0}\left([0, \tau] ; H^{3}(0, L)\right),
\end{array}\right.
$$


satisfying

$$
\left\{\begin{array}{lll}
y_{t}^{\alpha}-y_{x x}^{\alpha}+z^{\alpha} y_{x}^{\alpha}=p^{\alpha}(t) & \text { in } & (0, \tau) \times(0, L), \\
z^{\alpha}-\alpha^{2} z_{x x}^{\alpha}=y^{\alpha} & \text { in } & (0, \tau) \times(0, L), \\
z^{\alpha}(\cdot, 0)=y^{\alpha}(\cdot, 0)=v_{l}^{\alpha} & \text { on } & (0, \tau), \\
z^{\alpha}(\cdot, L)=y^{\alpha}(\cdot, L)=v_{r}^{\alpha} & \text { on } & (0, \tau) \\
y^{\alpha}(0, \cdot)=y_{0} & \text { in } & (0, L)
\end{array}\right.
$$

and, moreover,

$$
\left\|y^{\alpha}(\tau, .)-y_{f}\right\|_{H^{1}(0, L)} \leq K \sqrt{\tau}
$$

and

$$
\left\|p^{\alpha}\right\|_{C^{0}([0, T])}+\left\|\left(v_{l}^{\alpha}, v_{r}^{\alpha}\right)\right\|_{H^{3 / 4}\left([0, T] ; \mathbb{R}^{2}\right)} \leq C \quad \forall \alpha>0 .
$$

In order to prove this result, let us introduce $\lambda \in C_{0}^{1}((0,1) ;[0, \infty))$ with $\|\lambda\|_{L^{1}(0,1 / 2)}>L$ and $\lambda(t)=\lambda(1-t)$ for all $t \in[0,1]$. Let us set $\lambda^{\tau}(t):=\frac{1}{\tau} \lambda\left(\frac{t}{\tau}\right)$ for all $t \in[0, \tau]$.

The following two results hold:

Lemma 4.4. Let $M>0$ be a positive constant. Then, if $u_{0}, u_{f} \in C^{2}([0, L])$ and

$$
\max \left\{\left\|u_{0}\right\|_{C^{2}([0, L])},\left\|u_{f}\right\|_{C^{2}([0, L])}\right\} \leq M,
$$

there exists $\tau_{0} \in(0,1)$, independent of $\alpha$, such that for every $\tau \in\left(0, \tau_{0}\right]$ we can find controls $v_{l}^{\alpha, \tau}, v_{r}^{\alpha, \tau}$ in $C^{2}([0, \tau])$ and associated states $u^{\alpha, \tau}, w^{\alpha, \tau}$ in $C^{2}([0, \tau] \times([0, L]))$, satisfying

$$
\left\{\begin{array}{lll}
u_{t}^{\alpha, \tau}+\left(\lambda^{\tau}(t)+w^{\alpha, \tau}\right) u_{x}^{\alpha, \tau}=0 & \text { in }[0, \tau] \times[0, L], \\
w^{\alpha, \tau}-\alpha^{2} w_{x x}^{\alpha, \tau}=u^{\alpha, \tau} & \text { in }[0, \tau] \times[0, L], \\
w^{\alpha, \tau}(\cdot, 0)=v_{l}^{\alpha, \tau}, \quad w^{\alpha, \tau}(\cdot, L)=v_{r}^{\alpha, \tau} & \text { in }[0, \tau], \\
u^{\alpha, \tau}(\cdot, 0)=v_{l}^{\alpha, \tau} & \text { in } I_{l}, \\
u^{\alpha, \tau}(\cdot, L)=v_{r}^{\alpha, \tau} & \text { in } I_{r}, \\
u^{\alpha, \tau}(0, \cdot)=u_{0} & \text { in }[0, L], \\
u^{\alpha, \tau}(\tau, \cdot)=u_{f} & \text { in }[0, L],
\end{array}\right.
$$

where $I_{l}=\left\{t \in[0, T]: v_{l}^{\alpha, \tau}(t)>0\right\}$ and $I_{r}=\left\{t \in[0, T]: v_{r}^{\alpha, \tau}(t)<0\right\}$. Furthermore, there exists $C>0$, independent of $\alpha$ and $\tau$, such that

$$
\left\|u^{\alpha, \tau}\right\|_{C^{0}\left([0, \tau] ; C^{2}([0, L])\right)} \leq C M .
$$

Proof. First, thanks to the fact that $\|\lambda\|_{L^{1}(0,1 / 2)}>L$ and Remark 3.8, we know that there exists $\delta>0$ (independent of $\alpha$ ) such that, for any initial datum in a ball of $C^{2}([0, L])$ centered at origin and radius $\delta$, there exists a solution to $(3.2)$ belonging to $C^{2}([0,1 / 2] \times[0, L])$ satisfying $(3.20)$.

Let us now take $\tau_{0} \in(0,1)$ such that $\tau_{0} M \leq \delta$. Then, according to the previous construction, for each $\tau \in\left(0, \tau_{0}\right]$ there exist functions $\left.\left(\widetilde{y}^{\alpha}, \widetilde{z}^{\alpha}\right),\left(\widehat{y}^{\alpha}, \widehat{z}^{\alpha}\right) \in C^{2}\left([0,1 / 2] \times[0, L] ; \mathbb{R}^{2}\right)\right)$, solutions to (3.2) and satisfying $\widetilde{y}^{\alpha}(0, x)=\tau u_{0}(x)$ and $\widehat{y}^{\alpha}(0, x)=\tau u_{f}(L-x)$, for all $x \in[0, L]$, and (3.20).

Then, one defines the states

$$
u^{\alpha, \tau}(t, x):=\left\{\begin{array}{lll}
\tau^{-1} \widetilde{y}^{\alpha}\left(\tau^{-1} t, x\right) & \text { in } \quad[0, \tau / 2] \times[0, L] \\
\tau^{-1} \widehat{y}^{\alpha}\left(\tau^{-1}(\tau-t), L-x\right) & \text { in } \quad[\tau / 2, \tau] \times[0, L]
\end{array}\right.
$$


and

$$
w^{\alpha, \tau}(t, x):=\left\{\begin{array}{lll}
\tau^{-1} \widetilde{z}^{\alpha}\left(\tau^{-1} t, x\right) & \text { in } \quad[0, \tau / 2] \times[0, L] \\
\tau^{-1} \widehat{z}^{\alpha}\left(\tau^{-1}(\tau-t), L-x\right) & \text { in } \quad[\tau / 2, \tau] \times[0, L]
\end{array}\right.
$$

and the associated boundary controls

$$
v_{l}^{\alpha, \tau}(t):=w^{\alpha, \tau}(t, 0) \quad \text { and } \quad v_{r}^{\alpha, \tau}(t):=w^{\alpha, \tau}(t, L)
$$

Since $\lambda\left(\tau^{-1} t\right) \equiv \lambda\left(\tau^{-1}(\tau-t)\right)$, the couple $\left(u^{\alpha, \tau}, w^{\alpha, \tau}\right)$ belongs to the space $C^{2}\left([0, \tau] \times[0, L] ; \mathbb{R}^{2}\right)$ and satisfies (4.9) and (4.10).

Remark 4.5. Indeed, thanks to Remark 3.10, one sees that $u^{\alpha, \tau}$ and $w^{\alpha, \tau}$ coincides on the boundary, i.e. $u^{\alpha, \tau}(\cdot, 0)=w^{\alpha, \tau}(\cdot, 0)$ and $u^{\alpha, \tau}(t, L)=w^{\alpha, \tau}(t, L)$ in $[0, T]$.

Lemma 4.6. Assume that $M>0, u_{0}, u_{f} \in C^{2}([0, L])$ satisfy (4.8) and $\tau_{0}$ is furnished by Lemma 4.4. Then, there exists $\tau_{*} \in\left(0, \tau_{0}\right]$ such that, for any $\tau \in\left(0, \tau_{*}\right]$ and any $\left(u^{\alpha, \tau}, w^{\alpha, \tau}\right) \in C^{2}\left([0, \tau] \times[0, L] ; \mathbb{R}^{2}\right)$ satisfying $(4.9)$ and (4.10), there exists a unique solution to

$$
\left\{\begin{array}{lll}
r_{t}^{\alpha, \tau}+\left(q^{\alpha, \tau}+w^{\alpha, \tau}+\lambda^{\tau}\right) r_{x}^{\alpha, \tau}-r_{x x}^{\alpha, \tau}+q^{\alpha, \tau} u_{x}^{\alpha, \tau}-u_{x x}^{\alpha, \tau}=0 & \text { in }(0, \tau) \times(0, L), \\
q^{\alpha, \tau}-\alpha^{2} q_{x x}^{\alpha, \tau}=r^{\alpha, \tau} & \text { in }(0, \tau) \times(0, L), \\
r^{\alpha, \tau}(\cdot, 0)=0, \quad r_{x}^{\alpha, \tau}(\cdot, L)=0 & \text { in }(0, \tau), \\
q^{\alpha, \tau}(\cdot, 0)=0, \quad q^{\alpha, \tau}(\cdot, L)=r^{\alpha, \tau}(\cdot, L) & \text { in }(0, \tau), \\
r^{\alpha, \tau}(0, \cdot)=0 & \text { in }(0, L),
\end{array}\right.
$$

satisfying

$$
\left\{\begin{array}{l}
r^{\alpha, \tau} \in L^{2}\left(0, \tau ; H^{2}(0, L)\right) \cap H^{1}\left(0, \tau ; L^{2}(0, L)\right) \cap C^{0}\left([0, \tau] ; H^{1}(0, L)\right) \\
q^{\alpha, \tau} \in L^{2}\left(0, \tau ; H^{4}(0, L)\right) \cap H^{1}\left(0, \tau ; L^{2}(0, L)\right) \cap C^{0}\left([0, \tau] ; H^{3}(0, L)\right)
\end{array}\right.
$$

and

$$
\left\|r^{\alpha, \tau}\right\|_{L^{2}\left(0, \tau ; H^{2}(0, L)\right) \cap H^{1}\left(0, \tau ; L^{2}(0, L)\right)} \leq C .
$$

Here, $C$ is a positive constant that depends on $L, T, M$ and $\tau$, but it is independent of $\alpha$. Moreover, there exists a constant $K$ that depends on $L, T$ and $M$ (independent of $\alpha$ and $\tau$ ), such that

$$
\left\|r^{\alpha, \tau}\right\|_{C^{0}\left([0, \tau] ; H^{1}(0, L)\right)} \leq K \sqrt{\tau}
$$

Proof. The proof is standard. It can be easily obtained, for instance, via a Faedo-Galerkin technique in combination with well known energy estimates.

We can now achieve the proof of Proposition 4.3. Indeed, given $\tau \in\left(0, \tau_{*}\right]$, it is not difficult to see that

$$
\left(y^{\alpha}, z^{\alpha}\right):=\left(u^{\alpha, \tau}+r^{\alpha, \tau}+\lambda^{\tau}, w^{\alpha, \tau}+q^{\alpha, \tau}+\lambda^{\tau}\right)
$$

satisfies (4.5) and (4.6) with $p^{\alpha}(t)=\lambda_{t}^{\tau}$ and, thanks to Remark 4.5, boundary controls $v_{l}^{\alpha}(t)=u^{\alpha, \tau}(t, 0)+$ $r^{\alpha, \tau}(t, 0)+\lambda^{\tau}(t)$ and $v_{r}^{\alpha}(t)=u^{\alpha, \tau}(t, L)+r^{\alpha, \tau}(t, L)+\lambda^{\tau}(t)$. Moreover, using (4.9) ${ }_{6},(4.11)$ and the fact that $\lambda^{\tau}$ vanishes in the neighbourhood of $\tau$, we obtain (4.7). 


\subsection{Uniform local exact controllability to the trajectories}

The goal of this section is to prove the local exact controllability to space-independent trajectories for the Burgers- $\alpha$ system, with controls and associated states uniformly bounded with respect to $\alpha$ in appropriate spaces. Thus, let $\widehat{m} \in C^{1}([0, T])$ be given and note that $\left(\widehat{y}^{\alpha}, \widehat{z}^{\alpha}\right)=(\widehat{m}, \widehat{m})$ is a trajectory of viscous Burgers- $\alpha$ system with $\left(\widehat{p}^{\alpha}(t), \widehat{v}_{l}^{\alpha}(t), \widehat{v}_{r}^{\alpha}(t)\right)=\left(\widehat{m}^{\prime}(t), \widehat{m}(t), \widehat{m}(t)\right)$. We have the following result:

Theorem 4.7. Let $\alpha>0$ and $\widehat{m} \in C^{1}([0, T])$ be given. Then, there exists $\delta>0$ (independent of $\alpha$ ) such that for any initial data $y_{0} \in H^{1}(0, L)$ satisfying $\left\|y_{0}-\widehat{m}(0)\right\|_{H^{1}} \leq \delta$ there exist $p^{\alpha} \in C^{0}([0, T])$ and $\left(v_{l}^{\alpha}, v_{r}^{\alpha}\right) \in$ $H^{3 / 4}\left(0, T ; \mathbb{R}^{2}\right)$ and associated states $\left(y^{\alpha}, z^{\alpha}\right) \in L^{2}\left(0, T ; H^{2}\left(0, L ; \mathbb{R}^{2}\right)\right) \cap H^{1}\left(0, T ; L^{2}\left(0, L ; \mathbb{R}^{2}\right)\right)$ satisfying $(1.2)$ and

$$
y^{\alpha}(T, \cdot) \equiv \widehat{m}(T)
$$

Moreover, the following estimates hold:

$$
\left\|p^{\alpha}\right\|_{C^{0}([0, T])}+\left\|\left(v_{l}^{\alpha}, v_{r}^{\alpha}\right)\right\|_{H^{3 / 4}\left([0, T] ; \mathbb{R}^{2}\right)} \leq C \quad \forall \alpha>0
$$

where $C>0$ is a positive constant independent of $\alpha$.

Let us set $\left(y^{\alpha}, z^{\alpha}\right)=\left(u^{\alpha}+\widehat{m}, w^{\alpha}+\widehat{m}\right)$ and take $p^{\alpha}=\widehat{m}^{\prime}$. Then, $\left(u^{\alpha}, w^{\alpha}\right)$ must satisfy

$$
\begin{cases}u_{t}^{\alpha}-u_{x x}^{\alpha}+\left(w^{\alpha}+\widehat{m}\right) u_{x}^{\alpha}=0 & \text { in }(0, T) \times(0, L), \\ w^{\alpha}-\alpha^{2} w_{x x}^{\alpha}=u^{\alpha} & \text { in }(0, T) \times(0, L), \\ u^{\alpha}(\cdot, 0)=w^{\alpha}(\cdot, 0)=h_{l}^{\alpha} & \text { in }(0, T), \\ u^{\alpha}(\cdot, 0)=w^{\alpha}(\cdot, L)=h_{r}^{\alpha} & \text { in }(0, T), \\ u^{\alpha}(0, \cdot)=u_{0} & \text { in }(0, L),\end{cases}
$$

where $u_{0}:=y_{0}-\widehat{m}(0)$ and $\left(h_{l}^{\alpha}, h_{r}^{\alpha}\right):=\left(v_{l}^{\alpha}-\widehat{m}, v_{r}^{\alpha}-\widehat{m}\right)$. Therefore, Theorem 4.7 is equivalent to the local null-controllability to (4.14).

Proposition 4.8. Let the conditions of Theorem 4.7 be satisfied. There exists $\delta>0$ (independent of $\alpha$ ) such that, for any initial data $u_{0} \in H^{1}(0, L)$ satisfying $\left\|u_{0}\right\|_{H^{1}} \leq \delta$, there exist $\left(h_{l}^{\alpha}, h_{r}^{\alpha}\right) \in H^{3 / 4}\left(0, T ; \mathbb{R}^{2}\right)$ and $\left(u^{\alpha}, w^{\alpha}\right) \in$ $L^{2}\left(0, T ; H^{2}\left(0, L ; \mathbb{R}^{2}\right)\right) \cap H^{1}\left(0, T ; L^{2}\left(0, L ; \mathbb{R}^{2}\right)\right)$ satisfying (4.14) and

$$
u^{\alpha}(T, \cdot) \equiv 0
$$

Moreover, there exists a positive constant $C>0$ (independent of $\alpha$ ) such that

$$
\left\|\left(h_{l}^{\alpha}, h_{r}^{\alpha}\right)\right\|_{H^{3 / 4}\left([0, T] ; \mathbb{R}^{2}\right)} \leq C \quad \forall \alpha>0
$$

Proof. The proof of this result relies on a fixed-point argument. Thus, given $u_{0} \in H^{1}(0, L)$ and $\eta>0$, one can get by reflection method an extension $u_{0}^{*} \in H_{0}^{1}(-\eta, L+\eta)$, with

$$
\left\|u_{0}^{*}\right\|_{H_{0}^{1}(-\eta, L+\eta)} \leq \widehat{C}_{1}\left\|u_{0}\right\|_{H^{1}(0, L)}
$$

where $\widehat{C}_{1}$ is a positive constant independent of $\alpha$.

Let $R>0$ be given and consider the set

$$
B_{R}^{\eta}:=\left\{\bar{u} \in L^{\infty}\left(0, T ; C^{0}([-\eta, L+\eta]):\|\bar{u}\|_{L^{\infty}\left(0, T ; C^{0}([-\eta, L+\eta])\right)} \leq R\right\}\right.
$$


For any $\bar{u} \in B_{R}^{\eta}$, we can easily deduce that there exists a unique solution to

$$
\begin{cases}w-\alpha^{2} w_{x x}=\bar{u} 1_{(0, L)} & \text { in }(0, T) \times(0, L) \\ w(\cdot, 0)=\bar{u}(\cdot, 0), w(\cdot, L)=\bar{u}(\cdot, L) & \text { in } \quad(0, T) .\end{cases}
$$

Moreover, using the maximum principle, we obtain that

$$
\|w\|_{L^{\infty}\left(0, T ; C^{0}([0, L])\right)} \leq \widehat{C}_{2}\|\bar{u}\|_{L^{\infty}\left(0, T ; C^{0}([-\eta, L+\eta])\right)} \leq C R,
$$

where $\widehat{C}_{2}$ is a positive constant independent of $\alpha$.

Then, again using the reflection method, we get an extension $w^{*} \in L^{\infty}\left(0, T ; C^{2}([-\eta, L+\eta])\right)$ with

$$
\left\|w^{*}\right\|_{L^{\infty}\left(0, T ; C^{0}([-\eta, L+\eta])\right)} \leq \widehat{C}_{3}\|w\|_{L^{\infty}\left(0, T ; C^{0}([0, L])\right)} \leq \widehat{C}_{3} R
$$

where $\widehat{C}_{3}$ is a positive constant independent of $\alpha$.

We assume that $L<a<b<L+\eta$. Then, arguing as in the proof of Theorem 1 in [1], we find $v \in L^{\infty}((0, T) \times$ $(a, b))$ and $u \in L^{2}\left(0, T ; H^{2}(-\eta, L+\eta)\right) \cap L^{\infty}\left(0, T ; H_{0}^{1}(-\eta, L+\eta)\right)$ such that

$$
\left\{\begin{array}{lll}
u_{t}-u_{x x}+\left(w^{*}+\widehat{m}\right) u_{x}=v 1_{(a, b)} & \text { in } & (0, T) \times(-\eta, L+\eta), \\
u(\cdot,-\eta)=u(\cdot, L+\eta)=0 & \text { in }(0, T), \\
u(0, \cdot)=u_{0}^{*} & \text { in }(-\eta, L+\eta), \\
u(T, \cdot)=0 & \text { in }(-\eta, L+\eta),
\end{array}\right.
$$

and

$$
\|v\|_{L^{\infty}\left(0, T ; L^{\infty}(a, b)\right)} \leq C\left\|u_{0}\right\|_{H^{1}(0, L)}
$$

for some $C>0$ of the form

$$
C:=e^{C_{0}\left[1+1 / T+(1+T)\left(\left\|w^{*}\right\|_{L \infty}^{2}\left(L_{\infty}^{\infty}+\|\widehat{m}\|_{\infty}^{2}\right)\right]\right.} .
$$

where $C_{0}>0$ depends on $a, b, L$ and $\eta$ and independent of $\alpha$. Therefore, it is not difficult to deduce that the norm of $u$ in $H^{1}\left(0, T ; L^{2}(-\eta, L+\eta)\right), L^{2}\left(0, T ; H^{2}(-\eta, L+\eta)\right)$ and $L^{\infty}\left(0, T ; H_{0}^{1}(-\eta, L+\eta)\right)$ are bounded by $C\left\|u_{0}\right\|_{H^{1}}$, where $C$ is independent of $\alpha$.

Consequently, there exists $\delta>0$ (independent of $\alpha$ ) such that, if $\left\|u_{0}\right\|_{H^{1}} \leq \delta$, one has $\|u\|_{L^{\infty}\left(0, T ; C^{0}([-\eta, L+\eta])\right)} \leq R$ and the mapping $\Lambda_{\alpha}: B_{R}^{\eta} \mapsto B_{R}^{\eta}, \Lambda_{\alpha}(\bar{u}):=u$ is well defined. Note that

$-\Lambda_{\alpha}$ is well defined and continuous. Indeed, this follows from the uniqueness of solution of (4.17) and (4.18); the continuity is obtained by using standard parabolic estimates and the fact that, if $\bar{u}_{n} \rightarrow \bar{u}$ in $L^{\infty}\left(0, T ; C^{0}([-\eta, L+\eta])\right)$, then $w_{n}^{*} \rightarrow w^{*}$ in $L^{\infty}\left(0, T ; C^{0}([-\eta, L+\eta])\right)$ and, therefore, $u_{n} \rightarrow u$ in $L^{\infty}\left(0, T ; C^{0}([-\eta, L+\eta])\right)$.

- $F^{\eta}:=\Lambda_{\alpha}\left(B_{R}^{\eta}\right)$ is relatively compact in $L^{\infty}\left(0, T ; C^{0}([-\eta, L+\eta])\right)$. Indeed, one easily obtains that $F^{\eta}$ is bounded in $L^{\infty}\left(0, T ; H_{0}^{1}(-\eta, L+\eta)\right)$ and $F_{t}^{\eta}$ is bounded in $L^{2}\left(0, T ; L^{2}(-\eta, L+\eta)\right)$. Hence, applying again ([47], Cor. 4), we get the desired compactness.

Finally, by applying Schauder's Fixed-Point Theorem, we see that there exists $u \in B_{R}^{\eta}$ such that $\Lambda_{\alpha}(u)=u$. Then, the couple $\left(u^{\alpha}, v^{\alpha}\right)$, where $u^{\alpha}$ is the restriction to $(0, T) \times(0, L)$ of $u$ and $w$ is the solution to (4.17), belongs to $L^{2}\left(0, T ; H^{2}\left(0, L ; \mathbb{R}^{2}\right)\right) \cap H^{1}\left(0, T ; L^{2}\left(0, L ; \mathbb{R}^{2}\right)\right)$ and satisfies (4.14), (4.15) and (4.16) with controls $h_{l}^{\alpha}:=u(\cdot, 0)$ and $h_{r}^{\alpha}:=u(\cdot, L)$. 


\subsection{Global exact controllability}

In this section we prove Theorem 1.2 by combining the results obtained in Sections 4.1, 4.2 and 4.3. First recall that given $y_{0} \in L^{\infty}(0, L)$ and the unique associated solution $\left(y_{1}^{\alpha}, z_{1}^{\alpha}\right)$ to (4.1), Proposition 4.1 provides a time $T^{*} \in(0, T / 2)$ and a constant $M^{*}>0$ (both independent of $\alpha$ ) such that $y_{1}^{\alpha} \in C^{0}\left(\left[T^{*}, T\right] ; C^{2}([0, L])\right)$ and, moreover,

$$
\left\|y_{1}^{\alpha}\right\|_{C^{0}\left(\left[T^{*}, T\right] ; C^{2}([0, L])\right)} \leq M^{*}
$$

Now, let us fix $N \in \mathbb{R}$, let us set $M:=\max \left\{M^{*},|N|\right\}$ and assume that the constant $\tau^{*}>0$, furnished by Proposition 4.3 is small enough, such that $T^{*}<T / 2-\tau^{*}$. Then, $y_{2,0}^{\alpha}:=y_{1}^{\alpha}(T / 2-\tau, \cdot)$ belongs to $C^{2}([0, L])$ and, from (4.19) and Proposition 4.3, there exist $p_{2}^{\alpha} \in C^{0}([0, \tau]),\left(v_{l, 2}^{\alpha}, v_{r, 2}^{\alpha}\right) \in H^{3 / 4}\left(0, \tau ; \mathbb{R}^{2}\right)$ and associated states $\left(y_{2}^{\alpha}, z_{2}^{\alpha}\right) \in L^{2}\left(0, \tau ; H^{2}\left(0, L ; \mathbb{R}^{2}\right)\right) \cap H^{1}\left(0, \tau ; L^{2}\left(0, L ; \mathbb{R}^{2}\right)\right)$ satisfying (4.5), (4.6) and (4.7), with initial datum $y_{2,0}^{\alpha}$ and target $y_{f}=N$.

Finally, decreasing $\tau$ if necessary and setting $y_{3,0}^{\alpha}:=y_{2}^{\alpha}(\tau, \cdot)$, we deduce, thanks to (4.7), that $\left\|y_{3,0}^{\alpha}-N\right\|_{H^{1}} \leq$ $\delta$, where $\delta>0$ is the constant given in Theorem 4.7 for a control time $T / 2$. Hence, this theorem (applied with $\widehat{m} \equiv N)$, guarantees the existence of controls $\left(v_{l, 3}^{\alpha}, v_{r, 3}^{\alpha}\right) \in H^{3 / 4}\left(0, T / 2 ; \mathbb{R}^{2}\right)$ such that the associated states $\left(y_{3}^{\alpha}, z_{3}^{\alpha}\right)$ satisfying (1.2), (4.12) and (4.13), with $p^{\alpha} \equiv 0$ and initial datum $y_{3,0}^{\alpha}$.

To conclude, using $\left(y_{1}^{\alpha}, z_{1}^{\alpha}\right),\left(y_{2}^{\alpha}, z_{2}^{\alpha}\right)$ and $\left(y_{3}^{\alpha}, z_{3}^{\alpha}\right)$, and the associated controls, we can build the required solution, as stated in Theorem 1.2.

\section{Additional COMments And Questions}

\subsection{Controllability for Lipschitz-continuous data}

Proposition 3.3 and Theorem 1.1 also hold for $y_{0}$ in $C^{0,1}([0, L])$. Indeed, arguing as in the proof, one can guarantee that, as soon as the initial condition is small enough in the Lipschitz-continuous class, there exist $\left(v_{l}, v_{r}\right) \in C^{0,1}\left([0, T] ; \mathbb{R}^{2}\right)$ and an associated state $(y, z) \in C^{0,1}\left([0, T] \times[0, L] ; \mathbb{R}^{2}\right)$ satisfying (3.2) almost everywhere; furthermore, it is not difficult to check that all the estimates are uniform with respect to $\alpha$.

Note that this result improves Theorem 1 in [11].

\subsection{Passing to the limit as $\alpha \rightarrow 0$}

Thanks to Theorem 1.2, assuming that $y_{0} \in H_{0}^{1}(0, L)$, the family of controls $\left\{\left(p^{\alpha}, v_{l}^{\alpha}, v_{r}^{\alpha}\right)\right\}_{\alpha>0}$ of the viscous Burgers- $\alpha$ systems is uniformly bounded in $C^{0}([0, T]) \times H^{3 / 4}\left(0, T ; \mathbb{R}^{2}\right)$ (take into account Remark 3.11) and the associated family of states $\left\{y^{\alpha}\right\}_{\alpha>0}$ is uniformly bounded in the space $L^{2}\left(0, T ; H^{2}(0, L)\right) \cap H^{1}\left(0, T ; L^{2}(0, L)\right)$. It is not difficult to verify that $\left\{y^{\alpha}\right\}_{\alpha>0}$ converges, as $\alpha$ goes to 0 , to a controlled solution to the viscous Burgers equation with same initial datum $y_{0}$.

On the other hand, Theorem 1.1 establishes the existence of uniformly bounded controls for the non-viscous Burgers- $\alpha$ system; the family of associated solutions $\left\{y^{\alpha}\right\}_{\alpha>0}$ is uniformly bounded in $C^{1}([0, T] \times[0, L])$. Appropriate arguments like those presented in the last paragraph, used in combination with Remark 3.10, allow us to guarantee that $\left\{y^{\alpha}\right\}_{\alpha>0}$ converges, as $\alpha \rightarrow 0$, to a controlled solution to the non-viscous Burgers equation with the same initial datum $y_{0}$. For uncontrolled nonlocal conservation laws, a similar question related to the singular limit was studied in [14].

An additional interesting question is to determine the order of convergence of $y^{\alpha}$, in the convergence space.

\subsection{Null controllability with two controls}

In Theorems 1.1 and 1.2, we have used three scalar controls. It remains open to see whether, using arguments similar to those in [41], it is also possible to prove global uniform null controllability with only two scalar controls. 


\subsection{Global exact controllability to the trajectories}

At least two additional questions remain open here: (i) to obtain uniform global exact controllability to trajectories for the viscous Burgers- $\alpha$ system with trajectories in $W^{1, \infty}\left(0, T ; W^{1, \infty}\left(0, L ; \mathbb{R}^{2}\right)\right)$; (ii) to reduce the number of scalar controls.

\subsection{Less regular initial conditions}

In [41], the author proved a null controllability result for the viscous Burgers equation with initial datum in $L^{2}(0, L)$. Is it also possible to control uniformly $L^{2}$ initial conditions in the case of the viscous Burgers- $\alpha$ system?

\section{REFERENCES}

[1] F.D. Araruna, E. Fernández-Cara and D.A. Souza, On the control of the Burgers-alpha model. Adv. Differ. Equ. 18 (2013) 935-954.

[2] F.D. Araruna, E. Fernández-Cara and D.A. Souza, Uniform local null control of the Leray- $\alpha$ model. ESAIM: COCV 20 (2014) $1181-1202$.

[3] C. Bardos and U. Frisch, Finite-time regularity for bounded and unbounded ideal incompressible fluids using Hölder estimates, Proceedings of the conference held at the university of Paris-Sud Orsay, France (1975)., Vol. 565 of Lectures Notes in Math. Springer-Verlag (1976) 1-13.

[4] A.L. Bertozzi, J.B. Garnett and T. Laurent, Characterization of radially symmetric finite time blowup in multidimensional aggregation equations. SIAM J. Math. Anal. 44 (2012) 651-681.

[5] H.S. Bhat and R.C. Fetecau, A Hamiltonian regularization of the Burgers equation. J. Nonlinear Sci. 16 (2006) 615-638.

[6] H.S. Bhat, R.C. Fetecau and J. A. Goodman, Leray-type regularization for the isentropic Euler equations. Nonlinearity 20 (2007) 2035-2046.

[7] H.S. Bhat and R.C. Fetecau, Stability of fronts for a regularization of the Burgers equation. Quart. Appl. Math. 66 (2008) 473-496.

[8] H.S. Bhat and R.C. Fetecau, The Riemann problem for the Leray-Burgers equation. J. Differ. Equ. 246 (2009) $3957-3979$.

[9] H.S. Bhat and R.C. Fetecau, On a regularization of the compressible Euler equations for an isothermal gas. J. Math. Anal. Appl. 358 (2009) 168-181.

[10] R. Camassa and D.D. Holm, An integrable shallow water equation with peaked solitons. Phys. Rev. Lett. 71 (1993) $1661-1664$.

[11] M. Chapouly, Global controllability of nonviscous and viscous Burgers-type equations. SIAM J. Control Optim. 48 (2009) 1567-1599.

[12] A. Cheskidov, D. Holm, E. Olson and E. Titi, On a Leray- $\alpha$ model of turbulence. Proc. R. Soc. A 461 (2005) $629-649$.

[13] G.M. Coclite, K.H. Karlsen and Y.-S. Kwon, Initial-boundary value problems for conservation laws with source terms and the Degasperis-Procesi equation. J. Funct. Anal. 257 (2009) 3823-3857.

[14] M. Colombo, G. Crippa and L.V. Spinolo, On the singular local limit for conservation laws with nonlocal fluxes. Arch. Ratl. Mech. Anal. 233 (2019) 1131-1167.

[15] J.-M. Coron, Global asymptotic stabilization for controllable systems without drift. Math. Control Signals Syst. 5 (1992) $295-312$.

[16] J.-M. Coron, On the controllability of 2-D incompressible perfect fluids. J. Math. Pures Appl. 75 (1996) $155-188$.

[17] J.M. Coron and S. Xiang, Small-time global stabilization of the viscous Burgers equation with three scalar controls. J. Math. Pures Appl. 151 (2021) 212-256.

[18] K. Craig and A.L. Bertozzi, A blob method for the aggregation equation. Math. Comp. 85 (2016) $1681-1717$.

[19] A. Degasperis and M. Procesi, Asymptotic integrability, in Symmetry and Perturbation Theory, edited by A. Degasperis and G. Gaeta. World Scientific (1999) 23-37.

[20] J.I. Díaz, Obstruction and some approximate controllability results for the Burgers equation and related problems. Control of Partial Differential Equations and Applications. Vol. 174 of Lecture Notes in Pure and Appl. Math. Dekker, New York (1995) $63-76$.

[21] C.I. Doering and A.O. Lopes, Equações diferenciais ordinárias, 5 ed., Coleção matemática universitária, IMPA (2014).

[22] A. Doubova, E. Fernández-Cara, M. González-Burgos and E. Zuazua, On the controllability of parabolic systems with a nonlinear term involving the state and the gradient. SIAM J. Control Optim. 41 (2002) 798-819.

[23] J. Escher and Z. Yin, Initial boundary value problems for nonlinear dispersive wave equations. J. Funct. Anal. 174 (2009) 479-508.

[24] L. Evans, Partial differential equations, 2 ed., Vol. 19 of Graduate Studies in Mathematics. American Mathematical Society, Providence (2010).

[25] C. Fabre, J.-P. Puel and E. Zuazua, Approximate controllability of the semilinear heat equation. Proc. Roy. Soc. Edinburgh Sect. A 125 (1995) 31-61.

[26] E. Fernández-Cara and S. Guerrero, Null controllability of the Burgers system with distributed controls. Syst. Control Lett. 56 (2007) 366-372. 
[27] E. Fernández-Cara and D.A. Souza, Remarks on the control of a family of b-equations. Trends Control Theory Partial Differ. Equ. 32 (2019) 123-138.

[28] E. Fernández-Cara and E. Zuazua, Null and approximate controllability for weakly blowing up semilinear heat equations. Ann. Inst. H. Poincaré Anal. Non Linéaire 175 (2000) 583-616.

[29] C. Foias, D. Holm and E. Titi, The three dimensional viscous Camassa-Holm equation and their relation to the Navier-Stokes equation and turbulence theory. J. Dyn. Differ. Equ. 14 (2002) 1-36.

[30] C. Foias, C. Manley, O. Rosa and R. Temam, Navier-Stokes equations and turbulence. Vol. 83 of Encyclopedia of Mathematics and its Applications. Cambridge University Press (2001).

[31] A.V. Fursikov and O.Y. Imanuvilov, Controllability of evolution equations. Vol. 34 of Lecture Notes. Seoul National University, Korea (1996).

[32] O. Glass, Exact boundary controllability of 3-D Euler equation. ESAIM: COCV 5 (2000) 1-44.

[33] O. Glass and S. Guerrero, On the uniform controllability of the Burgers equation. SIAM J. Control Optim. 46 (2007) 1211-1238.

[34] S. Guerrero and O.Y. Imanuvilov, Remarks on global controllability for the Burgers equation with two control forces. Ann. Inst. H. Poincaré Anal. Non Linéaire 24 (2007) 897-906.

[35] J.K. Hale,Ordinary differential equations, 2nd edn. Krieger Press, Florida (1980).

[36] P. Hartman, Ordinary differential equations. John Wiley \& Sons (1964).

[37] D. Holm and M. Staley, Wave structure and nonlinear balances in a family of evolutionary PDEs. SIAM J. Appl. Dyn. Syst. 2 (2003) 323-380.

[38] T. Horsin, On the controllability of the Burgers equation. ESAIM: COCV 3 (1998) 83-95.

[39] J. Leray, Sur le mouvement d'un liquide visqueux emplissant l'espace. Acta Math. 63 (1934) 193-248.

[40] J.-L. Lions and E. Magenes, Vol. 2 of Non-Homogeneous Boundary Value Problems and Applications, Translated from the French by P. Kenneth. Die Grundlehren der Mathematischen Wissenschaften, Springer-Verlag, New York-Heidelberg (1972).

[41] F. Marbach, Small time global null controllability for a viscous Burgers' equation despite the presence of a boundary layer. $J$. Math. Pures Appl. 102 (2014) 364-384.

[42] G. Norgard and K. Mohseni, A regularization of the Burgers equation using a filtered convective velocity. J. Phys. A 41 (2008) 21.

[43] G. Norgard and K. Mohseni, On the convergence of the convectively filtered Burgers equation to the entropy solution of the inviscid Burgers equation. Multiscale Model. Simul. 7 (2009) 1811-1837.

[44] V. Perrollaz, Exact controllability of scalar conservation laws with an additional control in the context of entropy solutions. SIAM J. Control Optim. 50 (2012) 2025-2045.

[45] V. Perrollaz, Initial boundary value problem and asymptotic stabilization of the Camassa-Holm equation on an interval. $J$. Funct. Anal. 259 (2010) 2333-2365.

[46] C. Shen, On a regularization of a scalar conservation law with discontinuous coefficients. J. Math. Phys. 55 (2014) 15.

[47] J. Simon, Compact sets in the space $L^{p}(0, T ; B)$. Ann. Mat. Pura Appl. 146 (1987) 65-96. 